\title{
Formation of surface trailing counter-rotating vortex pairs downstream of a sonic jet in a supersonic crossflow
}

\author{
M. B. $\operatorname{Sun}^{1} \dagger$, Z. W. $\mathbf{H u}^{2}$ \\ ${ }^{1}$ Science and Technology on Scramjet Laboratory, National University of Defense Technology, \\ Changsha, 410073, China \\ ${ }^{2}$ Faculty of Engineering and the Environment, University of Southampton, Southampton, \\ SO17 1BJ, UK
}

(Received xx; revised xx; accepted $\mathrm{xx}$ )

Direct numerical simulations were conducted to uncover physical aspects of a transverse sonic jet injected into a supersonic crossflow at a Mach number of 2.7. Simulations were carried out for two different jet-to-crossflow momentum flux ratios $(J)$ of 2.3 and 5.5. It is identified that collision shock waves behind the jet induce a herringbone separation bubble in the near-wall jet wake and a reattachment valley is formed and embayed by the herringbone recirculation zone. The recirculating flow in the jet leeward separation bubble forms a primary TCVP (trailing counter-rotating vortex pair) close to the wall surface. Analysis on streamlines passing the separation region shows that the wing of the herringbone separation bubble serves as a micro-ramp vortex generator and streamlines acquire rotating momentum downstream to form a secondary surface TCVP in the reattachment valley. Herringbone separation wings disappear in the farfield due to the cross interaction of lateral supersonic flow and the expansion flow in the reattachment valley, which also leads to the vanishing of the secondary TCVP. A three-dimensional schematic of surface trailing wakes is presented and explains formation mechanism of the surface TCVPs.

Key words: supersonic flow, transverse sonic jet, direct numerical simulation, supersonic boundary layer, reattachment, trailing counter rotating vortices, collision shock

\section{Introduction}

A sonic jet in a supersonic crossflow is one of the canonical flows often used for fundamental research of turbulent mixing and combustion that has applications to scramjet engine combustors. It maintains many flow features of interest, such as the threedimensionality, shock structures, flow separation and recirculation, and wall-bounded free shear layer phenomenon. A significant body of work has been conducted, as summarized in the recent reviews by Karagozian (2010) and Mahesh (2013).

A typical flow topology of a transverse jet through an under-expanded sonic injection from a wall surface to a supersonic crossflow is illustrated in figure 1(a)-(b) (Dickmann \& Lu 2009; Viti et al. 2009). The flowfield involves complex 3D unsteady shocks, contact surfaces, shear layer and their interactions. The jet forms a major counter-rotating vortex

$\dagger$ Email address for correspondence: sunmingbo@nudt.edu.cn 


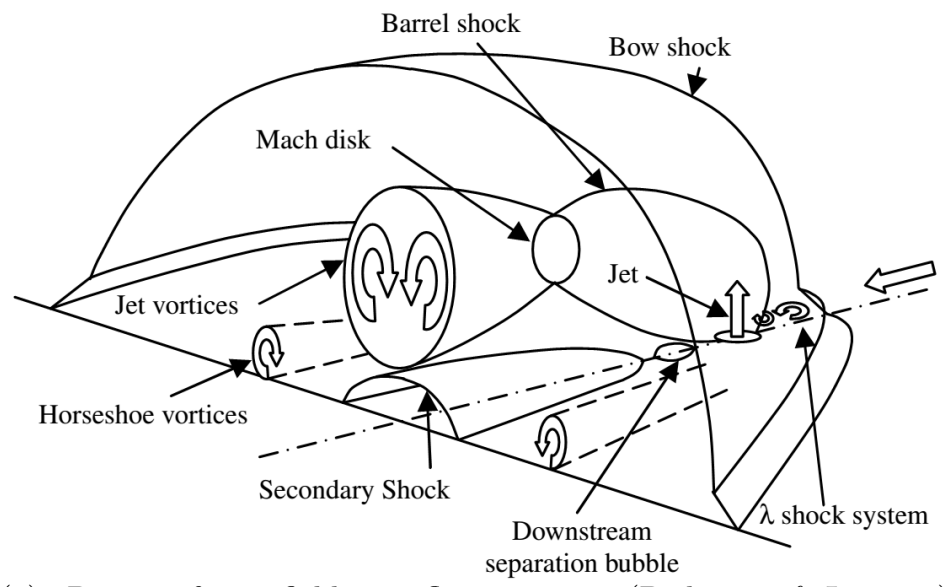

(a) 3D view of near-field meanflow structure (Dickmann \& Lu 2009)

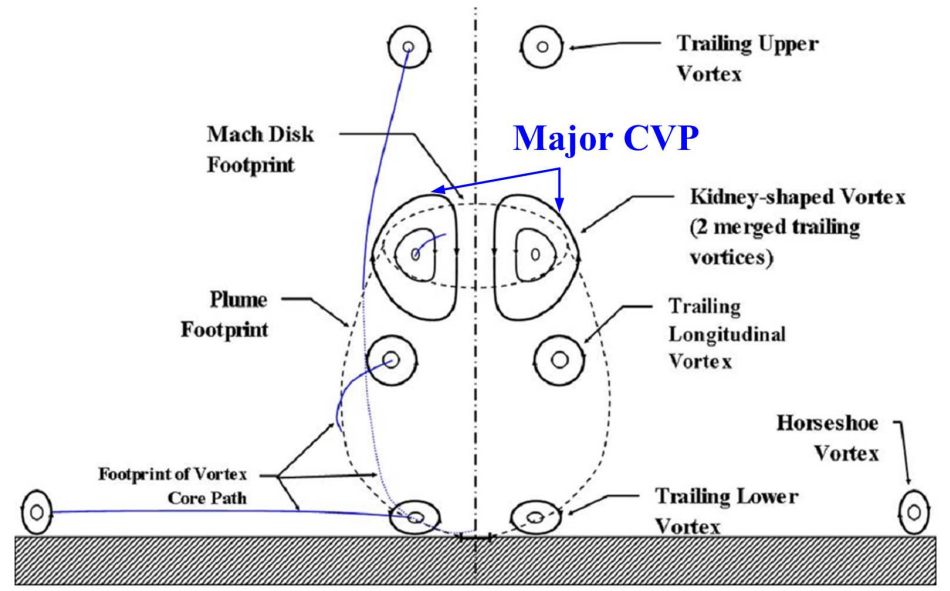

(b) front view of the flowfield aft of the barrel shock (Viti et al. 2009)

FiguRE 1. Schematic diagrams of a transverse jet in supersonic crossflow showing the dominant flow features

pair (CVP), whose axis is aligned with the streamwise direction and dominates the farfield mixing. A barrel shock around the plume terminating in a Mach disk and a bow shock is generated ahead of the jet. This causes boundary-layer separation upstream of injection and leads to the formation of a horseshoe vortex convecting around the jet. The most interesting part is about the wake structures in the jet leeward. As shown in figure 1(a), a local leeward separation bubble and a secondary shock is presented. The well-known lower trailing CVP is shown in figure 1(b) which has been depicted in many literatures.

Many experimental investigations have been conducted to understand the mechanisms of a jet injected into a supersonic crossflow, which include detailed velocity measurements (Santiago \& Dutton 1997), time-averaged wall-pressure measurements (Everett et al. 1998), the jet penetration height (Portz \& Segal 2006) and temporally-resolved flow visualizations (Gruber et al. 1997; Sun et al. 2013) and mixing characteristics (Ben-Yakar et al. 2006; Gamba \& Mungal 2015) with non-reactive and combustible gaseous jets. These measurements showed the overall jet flow features and the dynamics of the jet shear layer and shock interactions with boundary layers. Because of the difficulty in measuring 
the high-speed complex unsteady flowfields, experimental data are mostly limited to twodimensional (2D) slices of the flowfield for certain transverse and longitudinal planes.

Some numerical simulations have been conducted and the detailed flowfield and behavior of coherent structures have been investigated. Kawai \& Lele (2010) and Rana et al. (2011) performed large eddy simulations (LES) of a sonic jet in a supersonic crossflow and their results demonstrated Kelvin-Helmholtz $(\mathrm{K}-\mathrm{H})$ instabilities in the interface of jet shear layer confronted with the main stream. In their simulations, the jet near-field region is calculated with a jet-to-crossflow momentum ratio of $J=1.7$. Although the flowfield in the boundary layer was demonstrated, no further details were discussed on the jet wake flow. Won et al. Won et al. (2010) used detached-eddy simulation (DES) to reveal the vortex evolvement under the jet conditions of Ben-Yakar's experiment(BenYakar et al. 2006). Chai et al. (2015) conducted LES on flowfield of a sonic jet in a supersonic crossflow. In our previous work (Wang et al. 2013; Zhang et al. 2016), a hybrid RANS/LES methods were validated and used to study 3D jet mixing and the effect of micro-ramp on jet mixing in a supersonic crossflow. Recently, we conducted direct numerical simulations to study transverse jet in supersonic flows and focused on the upper trailing CVP (TCVP) which is located above the major CVP (Sun \& Hu 2018). The main conclusion was that the upper trailing CVPs are related to baroclinic effects caused by the deflecting Mach disk.

Combining experimental results with simulations, the shock structures and the jet vortices resulting from a sonic transverse jet injected into a supersonic crossflow have been well identified. In addition, formation mechanisms of the major CVP and the horseshoe vortices have also been well explained. In the near-wall jet wake flow, a TCVP is the generally agreed flow feature. Rana et al. (2011) discussed this TCVP and indicated that the TCVP is due to the low-pressure recirculation zone in the jet leeward and the suction action of the major CVP. The TCVP rotates in the opposite direction to major CVP and is dependent upon the free-stream Mach number. Kawai \& Lele (2010) inferred that TCVP is the pair of boundary-layer separation vortices along the symmetric plane induced by the suction of major CVP. Chai et al. (2015) presented inplane streamlines which reflected TCVPs but they did not give further discussion. Viti et al. (2009) described the flow features in details and deduced that TCVP originates from the second counter-rotating vortex of the upstream separation region and is energized by the shear layer of the barrel shock.

From the above analysis, it is found that there is no widely accepted conclusion on the formation mechanism of surface TCVP currently. Though many researchers referred to suction of the major CVP and separation, no works were done to mark separation domain and identify TCVP located in favorable or adverse flow. And paradox largely exists in the description of the flow structures from different literatures. For example, in figure 1 secondary shock is located downstream of the leeward separation and the possible separation bubble induced by the secondary shock is neglected. And it is still argued whether TCVP originates from the upstream separation (Viti et al. 2009) or the leeward separation (Rana et al. 2011). Furthermore, the detailed evolution of the TCVP in the farfield have not been investigated. Also it is important to know the near-wall jet wake to establish a clear physical understanding of the jet in supersonic crossflow. Experiments have not been able to demonstrate this, while RANS or LES cannot capture detailed turbulence behavior in the near-wall region. In the present work, DNS is conducted to investigate a sonic jet in a supersonic crossflow at $M a=2.7$ and to obtain the actual turbulence behind the jet near to the boundary layer. The significance of this investigation lies in the identification of separation topology and structures presented in supersonic boundary layer flow which have not been fully investigated by previous studies. 
This paper is organized as follows: the algorithm, flow conditions for the simulation, the computational grid, turbulent inflow generator and the validation of the numerical simulation are discussed in Section 2. The instantaneous jet wake flowfield is presented in Section 3.1. The mean flowfield of jet wakes, reattachment of the jet leeward separation and primary TCVP formation mechanism are presented in Section 3.2. Section 3.3 gives $3 \mathrm{D}$ streamlines which exhibit different types of local flow patterns related to the near-wall jet wakes, and further presents the mechanism of secondary TCVP.

\section{Flow conditions and numerical setup}

\subsection{Numerical simulation and inflow condition}

Simulations were run within an in-house code to solve the 3D unsteady compressible Navier-Stokes equations. The code has both direct and large eddy simulation capability and has been applied to many studies of instability, transition and turbulence in supersonic flows (Sandham et al. 2014; Sandham 2016) over the years. This paper only includes the main features of the code and explains the flow conditions. The detailed governing equations including the continuity, momentum and total energy equations for 3D flowfield can be found in Sandham et al. $(2014,2016)$.

The air inflow parameters (shown in Table 1) are set in accordance with the $M a=2.7$ experiments of Sun et al. (2013) with a stagnation pressure $P_{0}=101,325 \mathrm{~Pa}$, stagnation temperature $T_{0}=300 \mathrm{~K}$. The inflow $99 \%$ boundary-layer thickness, which is the same for all simulations, is estimated to be $\delta_{i}=5.12 \mathrm{~mm}$, with the compressible (including density variations) boundary-layer displacement and momentum thicknesses $\delta_{i}{ }^{*}=1.75 \mathrm{~mm}$ $\theta=0.38 \mathrm{~mm}$ and Reynolds numbers $R e_{\delta^{*}}=15,367, R e_{\theta}=3,337$. A sketch of the computational domain is shown in figure 2.

The injected air jet is modelled at two momentum flux ratios $J=2.3$ and $J=5.5$. Both cases lead to a sonic jet with a stagnation temperature $T_{0 i}=300 \mathrm{~K}$. The injection jet parameters are given in Table 2. Since the turbulent levels originating from the jet orifice are not known, a uniform profile across the jet orifice is implemented with no disturbance. It might be argued that turbulence would not be thoroughly resolved in the jet, however, it is believed that the overall behavior of coherent structures in jet and boundary layer will not be affected by this assumption.

\subsection{Domain and grid distribution}

To study jet interacting with a turbulent boundary layer, the incoming flow needs to be fully developed before the jet injection point. Although the use of a digital filter at the inflow boundary significantly reduces the length required for boundary-layer development (Xie \& Castro 2008), there should nevertheless be enough distance to allow the boundary layer to adjust to equilibrium upstream of the jet. It was found that a distance of 12 times the inflow boundary-layer thickness is enough to obtain realistic turbulent mean and rootmean-square (RMS) profiles from the digital filter inflow generator used. Accordingly, the initial length is set to be $L_{i} \approx 12.7 \delta_{i} \approx 65 \mathrm{~mm}$ for a fully developed turbulence generation (the region is denoted as STBL, supersonic turbulent boundary layer). The jet interaction length is given to be $L_{m}=90 \mathrm{~mm}$, including nearfield (denoted as JISC section1) with 10 $\mathrm{mm}$ upstream and $40 \mathrm{~mm}$ downstream of the jet and farfield (denoted as JISC section2) of $40 \mathrm{~mm}$. Therefore, the total length of the physical domain is $L_{x}=155 \mathrm{~mm}$. The wall-normal extension of the domain is $18 \mathrm{~mm}$, and a span of $14 \mathrm{~mm}$ is used. The jet centre is $75 \mathrm{~mm}$ downstream of the inlet with an orifice diameter of $D=2 \mathrm{~mm}$. The same computation domain was used in our previous work(Sun \& Hu 2018) and more details could be referred. 


$\begin{array}{lccccc}\text { Mach number } & \text { Stagnation } & \text { Stagnation } & \text { BL 99\% } & \text { Momentum } & \text { Reynolds } \\ & \text { temperature } & \text { pressure } & \text { thickness } & \text { thickness } & \text { number } \\ M a & T_{0} & P_{0} & \delta_{i} & \theta & R e_{\theta} \\ 2.7 & 300 \mathrm{~K} & 101 \mathrm{kPa} & 5.12 \mathrm{~mm} & 0.38 \mathrm{~mm} & 3337\end{array}$

TABLE 1. Air flow conditions for the simulations, including the dimensional boundary-layer (BL) thicknesses and Reynolds number at the air inflow

$\begin{array}{lcccc}\text { Jet-to-crossflow momentum } & \text { Mach number } & \text { Jet orifice } & \text { Stagnation } & \text { Stagnation } \\ \text { flux ratio } & & \text { diameter } & \text { temperature } & \text { pressure } \\ J & M a_{j} & D & T_{0 j} & P_{0 j} \\ 2.3 & 1.0 & 2 \mathrm{~mm} & 300 \mathrm{~K} & 138 \mathrm{kPa} \\ 5.5 & 1.0 & 2 \mathrm{~mm} & 300 \mathrm{~K} & 330 \mathrm{kPa}\end{array}$

TABLE 2. Air jet conditions for the simulations

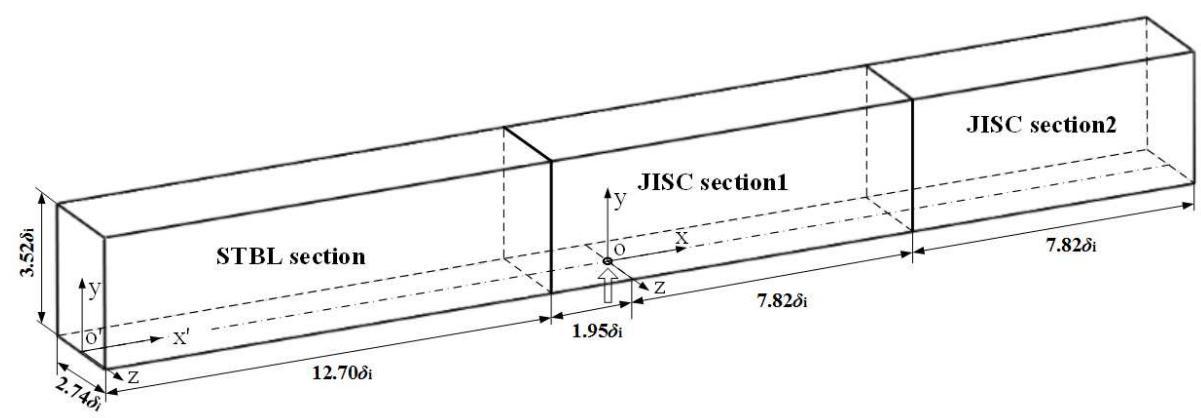

FIgURE 2. Schematic of the domain selected for the STBL (supersonic turbulent boundary layer) and JISC (jet interaction with a supersonic crossflow) simulations. Arrow indicates the jet centre, which is also the origin of the coordinate system.

Figure 2 shows the geometry of the physical domain, which is $155 \mathrm{~mm}\left(30.3 \delta_{i}\right)$, $18 \mathrm{~mm}\left(3.52 \delta_{i}\right), 14 \mathrm{~mm}\left(2.74 \delta_{i}\right)$ in the streamwise $(x)$, wall-normal $(y)$ and spanwise $(z)$ directions, respectively, for a Cartesian coordinate system with origin at the jet centre. A fine mesh of $2305 \times 279 \times 271$ grid points is used for the current simulations. The grid stretching scheme was described in our previous work(Sun \& Hu 2018) and for brevity the description is omitted. To capture the wall bounded flow, the grids are clustered near the wall in the region $y=0$ to $0.6 \delta_{i}$ uniformly and then stretched smoothly out. Table 3 summarizes the number of grid points and resolutions in wall units, which are calculated based on the friction velocity of the incoming turbulent boundary layer at $x=-15 \mathrm{~mm}$. In wall-normal direction, the grid has $y_{1}{ }^{+}=0.8$ for the first point off the wall and $\Delta_{y}^{+}$ $=5.2$ at the edge of the boundary layer and $\Delta_{y}^{+} \max _{10.0}$ in the mainstream. Since it is not known for the smallest turbulence scales in the jet field, the grid resolution of $\Delta_{y}^{+} \leqslant 10$ would ensure the mainstream simulations as quasi-DNS (QDNS), as denoted by Sandham et al. (2017). The uniform grids in the STBL and JISC2 regions have $\Delta_{x}^{+}=6.6$, while the JISC1 region has a resolution of $\Delta_{x}^{+}=4$.1. In the spanwise direction, grid is uniform with spacing fixed to $\Delta_{z}^{+}=4.1$. The fine mesh ensures good resolution of the near-wall turbulence. 


$\begin{array}{lccccccc} & N_{x} & N_{y} & N_{z} & \begin{array}{c}\text { Total } \\ \text { (million) }\end{array} & \Delta_{x}^{+} & \Delta_{y}^{+} & \Delta_{z}^{+} \\ \text {JISC scetion1 } & 945 & 279 & 271 & 74.5 & 4.1-6.6 & 0.8-10.0 & 4.1 \\ \text { JISC scetion2 } & 516 & 279 & 271 & 39.0 & 6.6 & 0.8-10.0 & 4.1 \\ \text { STBL scetion } & 774 & 279 & 271 & 58.5 & 6.6 & 0.8-10.0 & 4.1\end{array}$

TABLE 3. Computation grids for mesh of JISC and STBL domain

To minimize artificial reflections from boundaries, large buffer layers (not shown in figure. 2) with non-reflective outside-boundary set are introduced with lengths of $15 \mathrm{~mm}$, $11 \mathrm{~mm}$ and $12 \mathrm{~mm}$ at the outlet, on both the sides and the upper boundaries. The grid is smoothly stretched in the buffer layers towards the boundaries. The buffer layers with coarsened mesh help to damp fluctuations and minimize reflection from the boundaries.

No-slip and adiabatic boundary conditions are enforced on the bottom wall (shown in figure 2). An integrated characteristic boundary condition is applied at the inflow, and an outflow condition with an integrated characteristic scheme (Thompson 1987; Sandhu \& Sandham 1996) is applied in the spanwise boundary, top boundary and exit boundary, which are located on the edge of the buffer layer and largely decrease the numerical disturbances. The jet orifice is defined directly in the surface wall uniform grid and set to $J=2.3$ and $J=5.5$ with a sonic condition.

\subsection{Numerical methods and validation}

The governing equations are solved using a high-order finite-difference code (Sandham et al. 2002). This code uses a fourth-order central difference scheme for the spatial derivatives and a third-order explicit Runge-Kutta scheme for time advancement. The code makes use of an entropy splitting of Euler terms and Laplacian formulation of the viscous terms to improve the stability of the non-dissipative central scheme. In addition, a variant of the standard total variation diminishing scheme is used for shock capturing (Yee et al. 1999). To better resolve the steep gradient in flowfield around the injected jet, we used a hybrid scheme which combines the entropy splitting central scheme with a central-upwind WENO-CU4 scheme, which is developed following the procedure of the WENO-CU6 (Hu et al. 2010) and given in our recent work (Sun \& Hu 2018). The two schemes are combined using the Ducros sensor (Ducros et al. 1999) as the hybriding function. This hybrid scheme was well described in the paper (Sun \& Hu 2018) and for brevity omitted here.

The digital filter inflow generation method of Xie \& Castro (2008) is used to generate a more realistic supersonic turbulent boundary layer at the inflow. A detailed description of the optimized digital filter used here can be found in Touber (2010). The digital filter is robust to the choice of length scale, as long as the prescribed length scales are at least as large as the integral length scales of the real flow and sufficient downstream distance is provided to allow for the turbulence to develop (Wang et al. 2015b; Sun \& Hu 2018). The mean inflow profile is generated using the same approach as Touber \& Sandham (2009). The inflow RMS values are taken from the DNS results of Schlatter \& Orlu (2010) for a similar Reynolds number. A compressible scaling, as suggested by Morkovin (1962) and validated by Duan et al. (2011) for high Mach numbers, is used to transform the incompressible RMS values.

Statistical data are based on averaging flowfields over 100 non-dimensional time units $\left(D / U_{\infty}\right)$ after running the simulations for 200 non-dimensional units to let the flow 


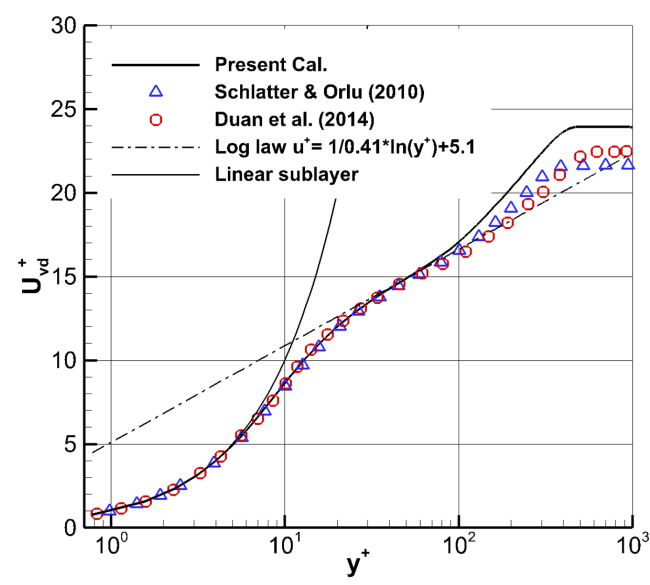

(a) Mean velocity distribution

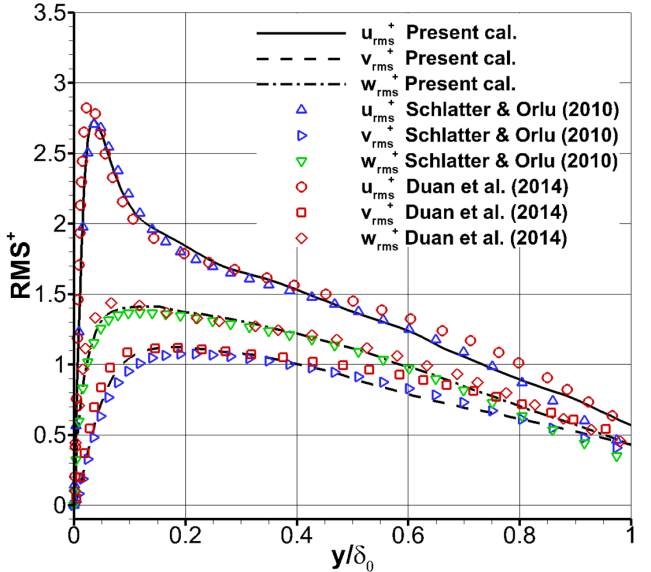

(b) RMS velocity distribution

FIgURE 3. Mean (a) and RMS (b) velocity distributions obtained at $60 \mathrm{~mm}$ downstream of the inlet. The van Driest transformation was applied to the mean velocity and a Morkovin scaling $\xi=\sqrt{\rho / \rho_{w}}$ to the RMS curves.

develop. The boundary layer thickness at the beginning the JISC section1 (at $x=-10 \mathrm{~mm}$ ) ahead of the injection is calculated to be $\delta g g \approx 5.64 \mathrm{~mm}$ based on a developed boundary layer without jet and the corresponding friction Reynolds number is estimated to be $R e_{\tau} \approx 8737$. Injection of $J=2.3$ case has a little influence on the boundary layer flow at $x$ $=-10 \mathrm{~mm}$ ahead of the jet. Thus turbulent boundary-layer mean profiles and distributions of RMS values at $x=-15 \mathrm{~mm}$, which has not been affected, are first evaluated. Here the coordinate origin is at the jet center and this position corresponds to $x / D=-7.5$. The calculated compressible boundary-layer displacement and momentum thicknesses at $x / D=-7.5$ is $\delta_{0}{ }^{*}=2.12 \mathrm{~mm}, \theta_{0}=0.41 \mathrm{~mm}$, respectively. It should be noted that the Reynolds number based on the freestream flow properties and the momentum thickness, i.e., $\operatorname{Re}_{\theta}=\rho_{e} U_{e} \theta / \mu_{w}$, is 3,600 . The corresponding Reynolds number under the van Driest scaling, i.e., $\operatorname{Re}_{\theta, v d}=\rho_{w} U_{e}^{v d} \theta^{v d} / \mu_{w}$, is 917 . The subscripts ' $e$ ' and ' $w$ ' above denote the main flow and wall parameters, respectively, while the superscript ' $v d$ ' means the value under the van Driest transform. Figure 3 shows the mean and RMS values collapsing very well with the DNS results of Schlatter \& Orlu (2010) with Reynolds number of 1,000, as also found in the recent study(Sun \& Hu 2018). DNS data of supersonic boundary layer from Duan et al. (2014) are compared in figure 3 and it is found that the current calculation also agrees well with the compressible velocity profile and the Reynolds-stress data with $M a_{\infty}=2.5$ and $R e_{\theta}=2835$.

In the previous study(Sun et al. 2013), the jet penetration height correlation was obtained from the statistics of NPLS (nanoparticle-based planar laser scattering) experimental images by using an upper edge approximation definition,

$$
\frac{y}{D J}=\frac{2.933}{J^{0.5830}}\left(\frac{x}{D J}\right)^{0.161}
$$

where $x$ represents the distance to jet orifice and $y$ represents the nomal distance to the wall. Gruber et al. (2000) suggested a power law fit based on jet concentration identification; however Ben-Yakar et al. (2006) and Sun et al. (2013) found an obvious deviation from their experiments. As discussed in the previous study(Ben-Yakar et al. 

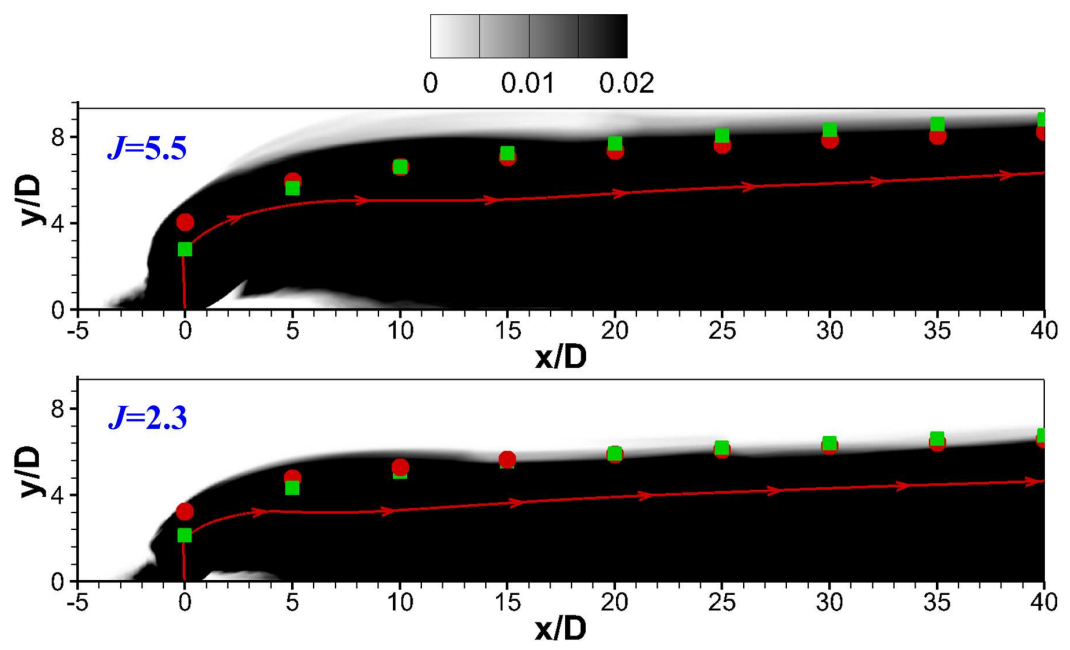

Figure 4. Penetration of the jet in the supersonic crossflow displayed by time averaged passive scalar contours, • represents data from penetration correlation (2.1), represents data from penetration correlation (2.2), streamlines originate from the centre of the jet exit.

2006; Sun et al. 2013), better agreement was achieved with the correlation of Rothstein \& Wantuck (1992),

$$
\frac{y}{D J}=J^{-0.6985} \ln \left[4.704 J\left(\frac{x}{D J}+\frac{0.6373}{J}\right)\right]
$$

A transport equation for a passive scalar of jet fluid is solved in the simulation. Figure 4 shows the contours of time averaged passive scalar for $J=5.5$ and $J=2.3$. The approximate penetration boundary based on a concentration identification is obtained and compared with the calculated data from (2.1) and (2.2). It is seen that the current penetration result agrees well with previous experimental correlations for both $J=5.5$ and $J=2.3$.

Similar to Muppidi \& Mahesh (2005) and Chai et al. (2015), the jet trajectory in the present study is defined as the streamline originating from the centre of the jet exit (centre streamline) on the time-averaged symmetry plane. In figure 4, the jet trajectory of $J=5.5$ and $J=2.3$ is extracted and shown from the velocity field respectively. Schetz \& Billig (1966) analysed a sonic jet in a supersonic crossflow $\left(M_{\infty}=2.1-2.7\right)$ at $J=4.75-10$, and derived a differential equation that can be used to predict the jet trajectory,

$$
\frac{d \alpha}{d \bar{s}}=-\frac{C_{D}(\alpha) \sin ^{2}(\alpha)}{2.5 \pi} \frac{1}{J}\left(\frac{\rho}{\rho_{j}}\right)(2.25+0.22 \bar{s})^{3}
$$

where $\bar{s}=s / d$ and $C_{D}$ is a drag coefficient determined by

$$
C_{D}=\left\{\begin{array}{lc}
1.2+\left(M_{\infty} \sin \alpha\right)^{7 / 2} & 0 \leqslant M_{\infty} \sin \alpha \leqslant 1 \\
1.06+1.14\left(M_{\infty} \sin \alpha\right)^{-3} & M_{\infty} \sin \alpha \geqslant 1
\end{array}\right.
$$

The jet trajectory can be obtained by integrating the Equation (2.3) from $\pi / 2$ to 0 . Schetz \& Billig (1966) further assumed that $\rho$ does not change along the jet, i.e. $\rho=\rho_{j}$. According to the suggestion of Chai et al. (2015), the density variation along the jet (dashed-dotdotted line in figure 5) should be taken into account. Chai et al. (2015) defined an effective density ratio $\left(\rho / \rho_{j}\right)^{e f f}=\rho_{f} / \rho_{b} . \rho_{b}$ is the density of the jet immediately behind the barrel shock, which is the effective jet exit where the jet actually 


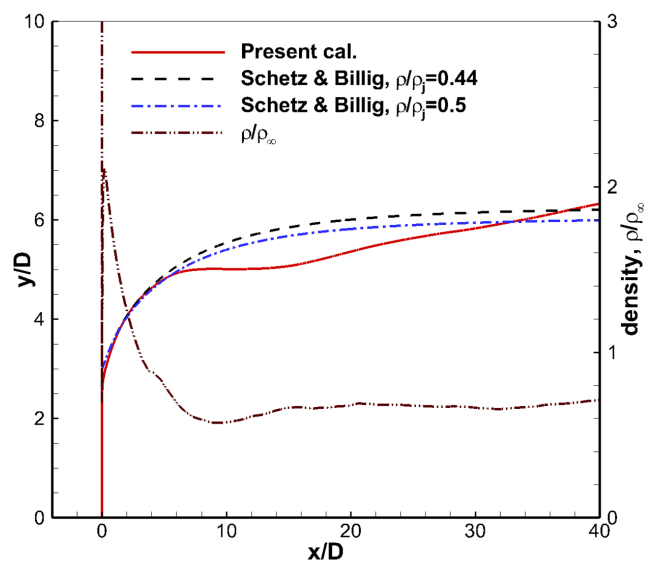

(a) $J=5.5$

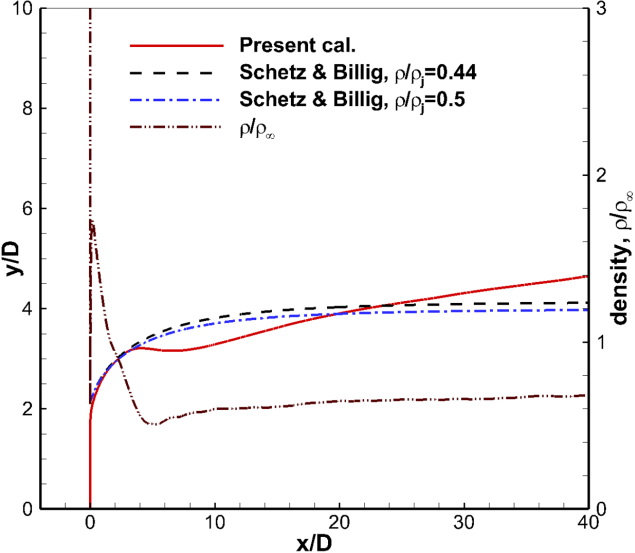

(b) $J=2.3$

Figure 5. Comparison between present calculation of jet trajectory and prediction using modified theory of Schetz \& Billig (1966).

sees the crossflow; and $\rho_{f}$ is the average density far downstream of the jet. The jet density variation for $J=5.5$ is that $\rho_{b}=1.39$ and $\rho_{f}=0.61$, which results in $\left(\rho / \rho_{j}\right)^{\text {eff }} \approx 0.44$. Further, we note that after interaction with the barrel shock, the centre streamline turns suddenly by approximately $30^{\circ}$. Therefore, (2.3) should be integrated from $60^{\circ}$ instead of $90^{\circ}$. (2.3) predicts the trajectory of $J=5.5$ marked by dashed-dotted curve in figure 5 (a). Chai et al. $(2015)$ considered $\left(\rho / \rho_{j}\right)^{\text {eff }} \approx 0.5$, which was also given in figure $5(\mathrm{a})$. These results shows reasonably good agreement with the current simulated result in the near field, and some deviation in the far field. Figure 5(b) shows that the jet trajectory for $J=2.3$, in which the prediction of Schetz \& Billig (1966) deviates the calculated jet penetration more clearly in the far field than $J=5.5$. It is inferred that (2.3) exhibits larger errors from simulations when smaller $J(<4.75)$ is conducted.

\section{Numerical results and analysis}

All simulations were performed on Archer, UK National Supercomputer. Each simulation requires approximately 540,000 processor core hours for flow development and collecting statistics over 100 non-dimensional time units $\left(D / U_{\infty}\right)$.

\subsection{Instantaneous flow structures in jet nearwall wakes}

Typical instantaneous results of $\lambda_{2}$ iso-surface(Jeong \& Hussain 1995) in the jet flowfield of $J=5.5$ and $J=2.3$ are shown in figure 6 , colored by the local density. It shows clearly the mixing process of the low density jet plume with the main stream and the incoming boundary layer. Jet plume density is low in the near-field and increases as the jet plume is convected downstream. The sonic jet with higher $J(=5.5)$ has a more intense interaction with the incoming flow, which results in a larger separation region and a higher penetration height. Typical instantaneous simulated results of density contours at $z / D=0.0$ in the flowfield of $J=5.5$ and $J=2.3$ are shown in figure 7 , which also corresponds to the density variation in figure 5 . In particular the shock and turbulent structures are well-identified. The jet structures, including the bow shock, the barrel shock and separation bubbles and the horseshoe vortex have been analyzed in details in previous literatures and we will focus on jet wake flows.

In figure 7 it is seen that twisted envelope shock appears behind the jet in both 


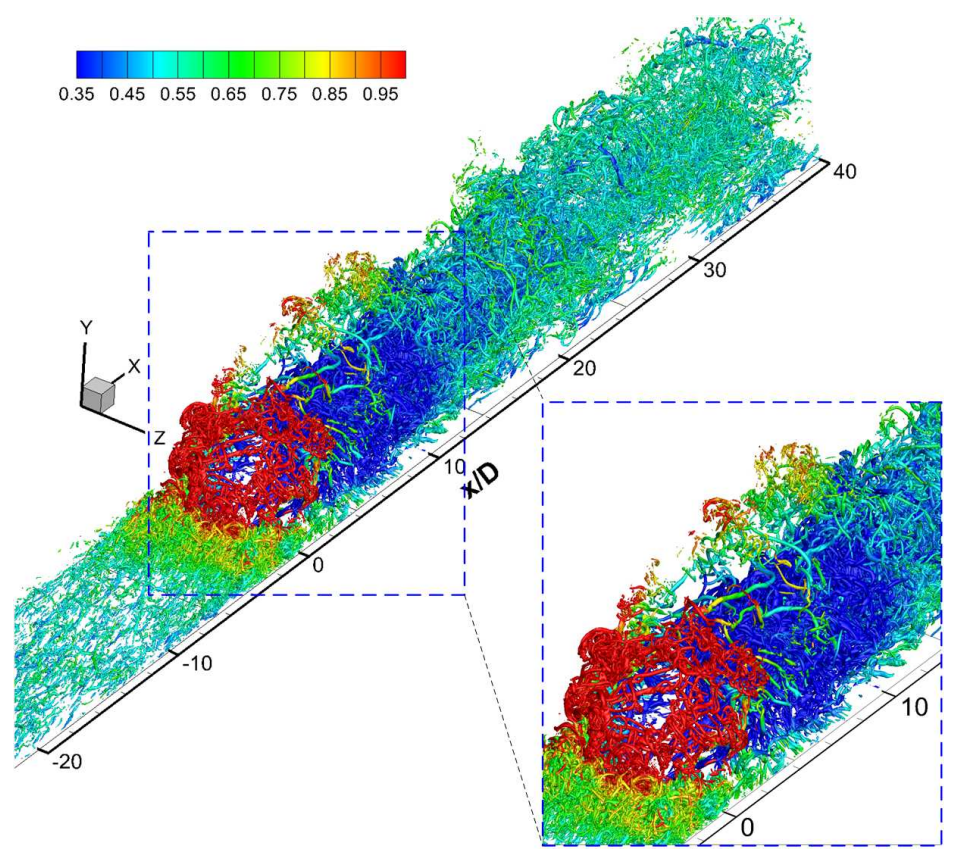

(a) $J=5.5$

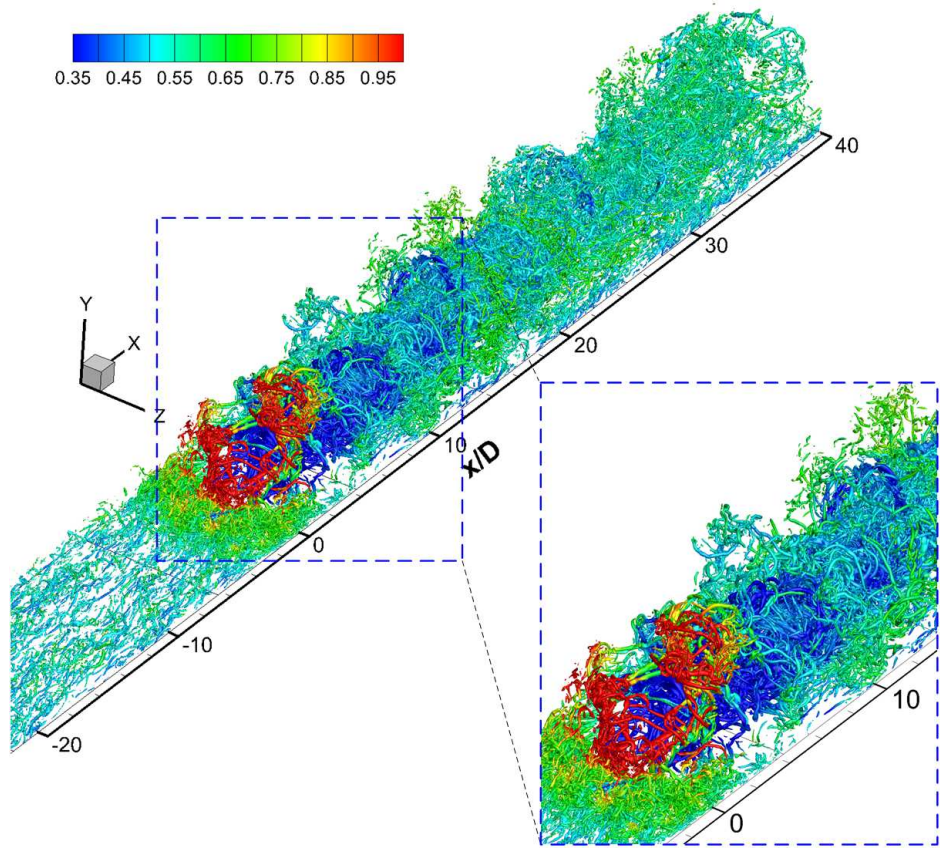

(b) $J=2.3$

Figure 6. Instantaneous iso-surfaces of vortex structures $\left(\lambda_{2}=-0.3\right)$ at $J=5.5$ and $J=2.3$ colored by local density 


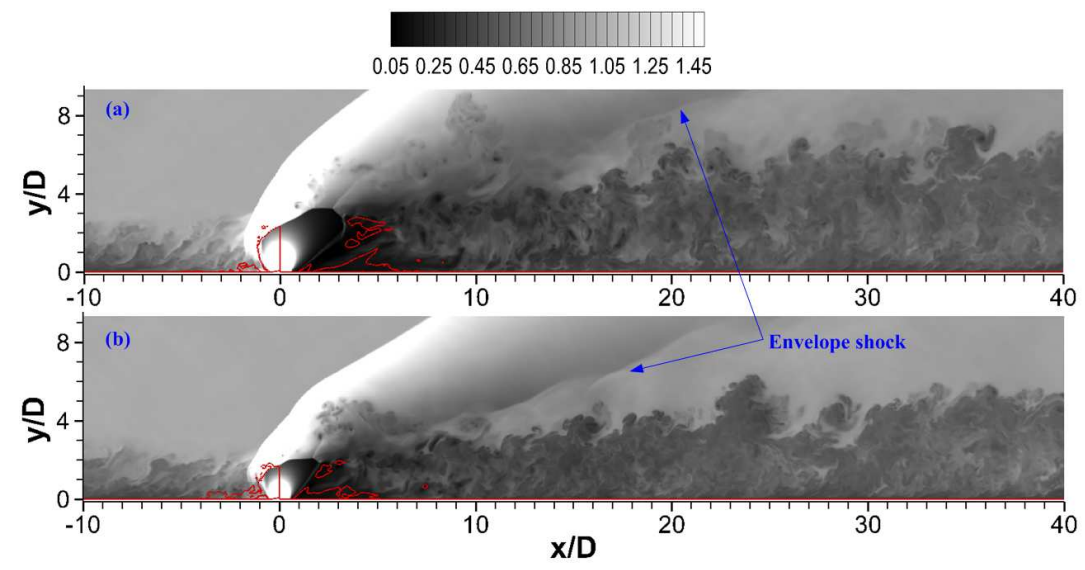

FiguRE 7. Density contour at $z / D=0.0$ slice of jet interaction with supersonic crossflow, (a) $J=5.5$, (b) $J=2.3$, isoline of $u / U_{\infty}=0.0$ is superimposed in red color

cases and it is clearer in $J=2.3$ case. As described in many literatures (Mahesh 2013; Morkovin et al. 1952), the envelope shock originates from the pressure gradients around the expanding jet. A strong mixing occurs in the jet wake flow and turbulent structures are identical in both the the near-field and the far-field. Near-wall density fields are shown in figure 8 on horizontal planes. Turbulent streaks are exhibited in figure $8(\mathrm{a})$-(b) at $y / D=0.08$, which reflect the local turbulence, and their response to the jet injection will be analyzed in another paper. There appear herringbone separation zones (wrapped by solid lines representing zero streamwise velocity) which extends both in streamwise and spanwise direction, as shown in figure 8 at $y / D=0.08$ slices. In the lateral downstream of the jet orifice, local density magnitude decreases significantly, which implies that expansion occurs near the jet leeward. High velocity fluid moves downstream around the jet and interacts with the shock generated by the collision of the flow around the jet barrel. The collision shock waves are clearly seen in density contours on $y / D=1.0$ slice of $J=5.5$ and $y / D=0.75$ slice of $J=2.3$ and they are twisted due to local turbulence. It is clear that these collision shocks induce the herringbone separation in the near-wall region and they have a complex interaction. The collision shock is $3 \mathrm{D}$ since the separation zone is herringbone and there are two separation oblique wings. In figure 8(a)-(b), it is seen that the herringbone separation region is confined and flow reattaches downstream of the cross point of the wings. In the vicinity of the symmetric plane, a recovery region is embayed by the herringbone separation region near the centerline. The herringbone separation region for $J=5.5$ is larger than the $J=2.3$ case and their flow patterns are similar.

\subsection{Mean flow properties in the jet nearwall wake flowfield}

Time-averaged density contours at $z / D=0.0$ slice are shown in figure 9 . It is seen that the averaged flow patterns are similar for $J=5.5$ and 2.3. The penetration height, barrel shock size and jet leeward separation size of $J=5.5$ is larger than $J=2.3$. The envelope shock related to expanding jet barrel is clear on time-averaged contour. Skin friction coefficient contours are shown at $y / D=0.0$ in figure $10(\mathrm{a})$-(b). The solid isolines superimposed on the filled contours correspond to $u / U_{\infty}=-0.0001$ and the region surrounded by the isolines represents a separated zone. It clearly shows that the mean herringbone separation region is limited in the cramp of the collision shock waves. In the vicinity of the symmetric plane, flow reattaches more quickly than outside, resulting in a $\mathrm{V}$-shape 


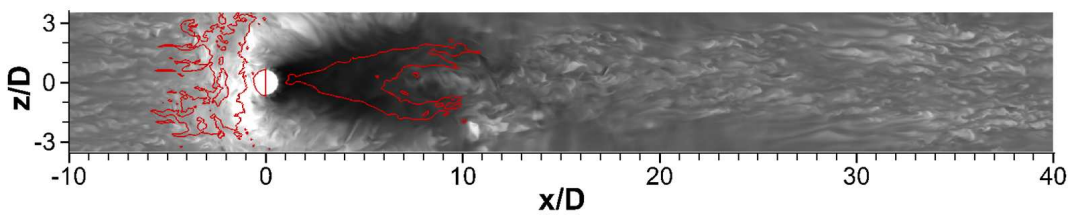

(a) density contour at $y / D=0.08$ slice of $J=5.5$

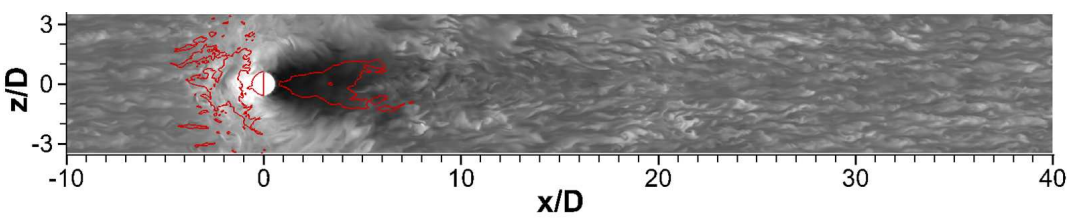

(b) density contour at $y / D=0.08$ slice of $J=2.3$

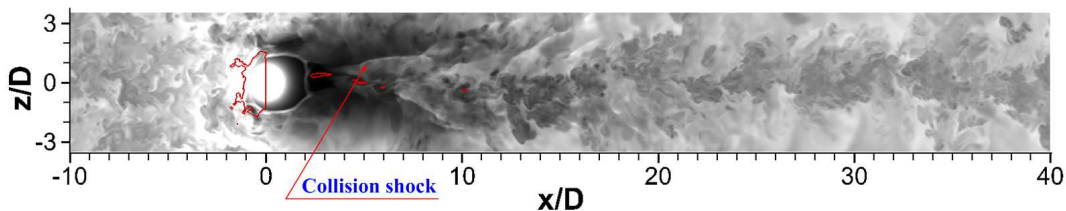

(c) density contour at $y / D=1.0$ slice of $J=5.5$

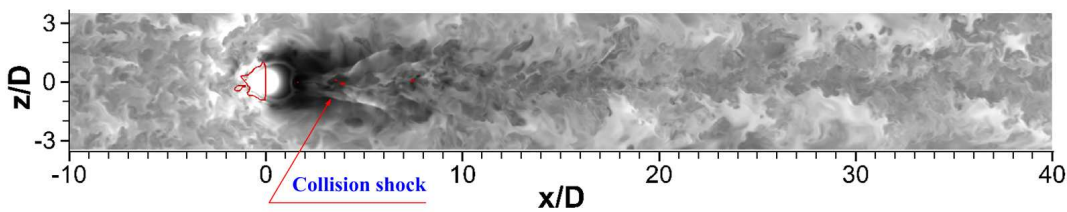

(d) density contour at $y / D=0.75$ slice of $J=2.3$

FiguRE 8. Instantaneous streamwise velocity streaks at different horizontal planes of jet interaction with the incoming boundary layer for $J=5.5$ and $J=2.3$ cases, isolines of $u / U_{\infty}=0$ are superimposed

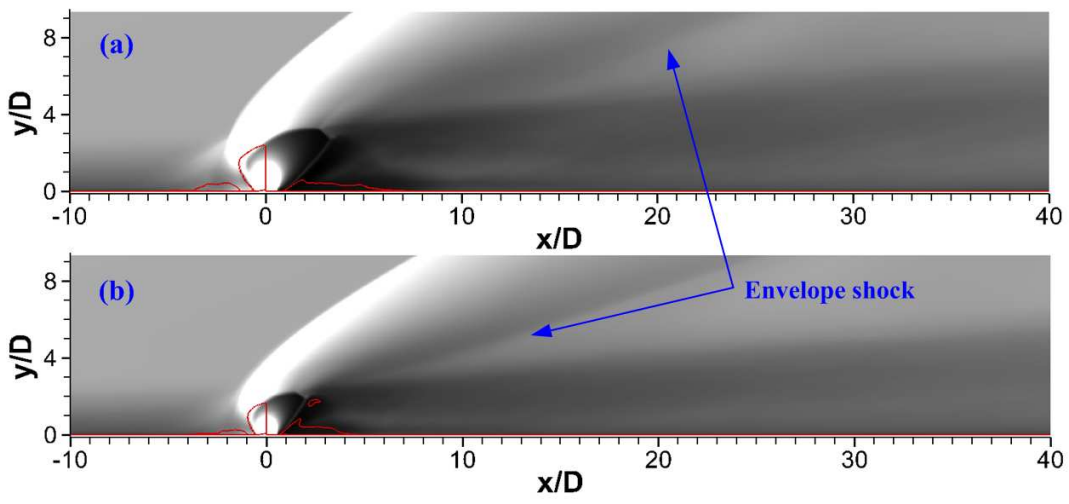

Figure 9. Time-averaged density contours on $z / D=0.0$ plane for (a) $J=5.5$ and (b) $J=2.3$ case, isolines of $u / U_{\infty}=0.0$ are superimposed

reattachment line (as shown in figure 10a) behind the separation zone. The herringbone separation size of $J=5.5$ is larger than $J=2.3$ and it is expected that the maximum width of the separation is related to $J$. The interesting part is the half-span expanding angle of the mean separation line (drawn by a solid line in figure 10(a)-(b)) with jet centerline. 


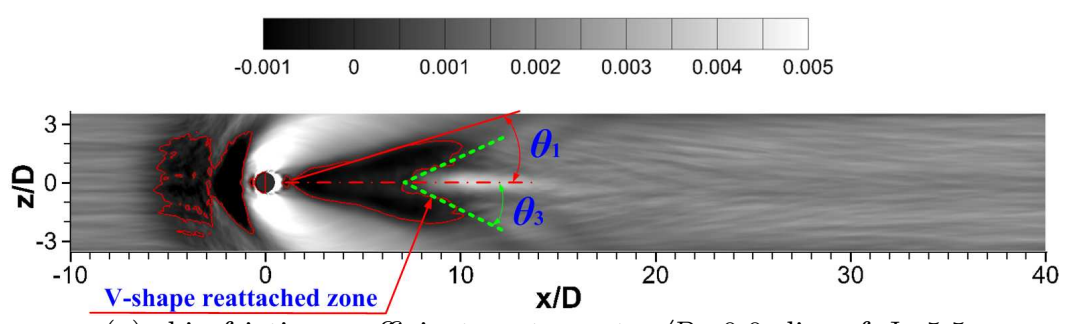

(a) skin friction coefficient contour at $y / D=0.0$ slice of $J=5.5$

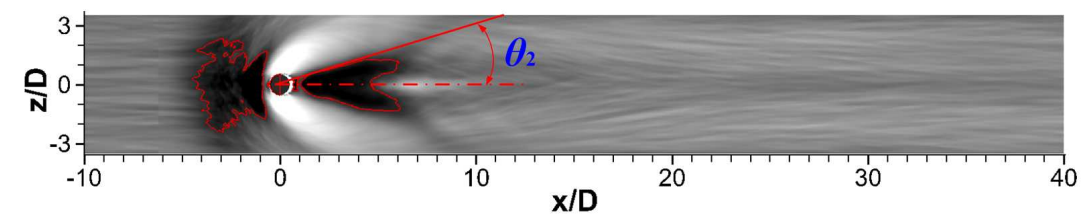

(b) skin friction coefficient contour at $y / D=0.0$ slice of $J=2.3$

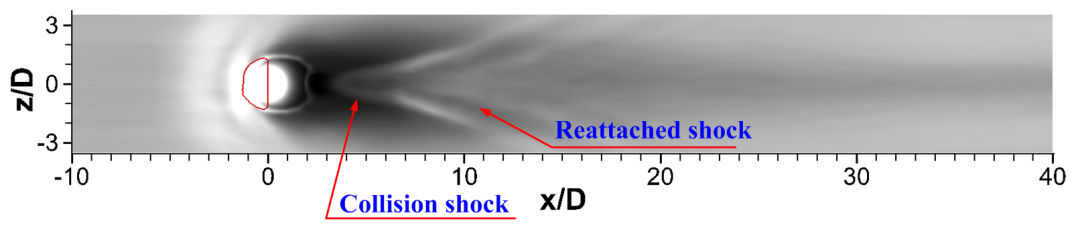

(c) density contour at $y / D=1.0$ slice of $J=5.5$

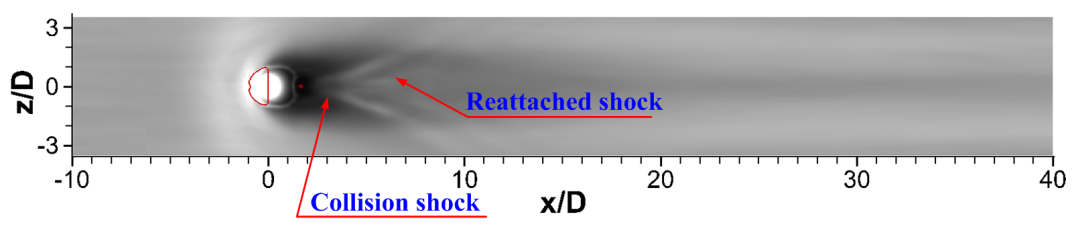

(d) density contour at $y / D=0.75$ slice of $J=2.3$

FiguRE 10. Time-averaged skin friction coefficient and density contours on different horizontal planes for $J=5.5$ and $J=2.3$ case, isolines of $u / U_{\infty}=0.0$ are superimposed

A comparison between $J=2.3$ case and $J=5.5$ case shows that the expanding of the separation region is virtually the same for $J=5.5$ (figure $10 \mathrm{a}$ ) and $J=2.3$ (figure $10 \mathrm{~b}$ ), that is, $\theta_{1} \approx \theta_{2} \approx 15.6^{\circ}$. This feature demonstrates that the separation line is independent of $J$. This could be expected since separation is mainly induced by the leeward collision shock and collision shock angle is only dependent upon the free-stream Mach number. The increase of $J$ or orifice diameter would not change the collision shock angle and the half-span expanding angle of separation line. The deflection angle $\theta_{3}\left(\approx 28.0^{\circ}\right)$ of the $\mathrm{V}$-shape reattachment line (shown in figure10a) with the jet centerline is larger than $\theta_{1}$ and $\theta_{2}$. In figure $10(\mathrm{c})-(\mathrm{d})$, time-averaged collision shock waves and reattached shock waves are identified. Reattached shock locates behind the collision shock and between them exists a low density field, which reflects local expansion and reattachment in separation/shock interaction. As discussed in Section 3.1, reattached shock waves reveal the three-dimensionality of the herringbone separation zone.

Figure 11 represents the streamlines on the cross flow planes downstream of the jet plume for $J=2.3$ and $J=5.5$, respectively. The major CVP appears due to the interaction of the free-stream flow with the jet fluid and grows in size further downstream. As the major CVP starts to grow in size as in figure 11 another small pair of vortices, named 
lower trailing CVP, occurs near to surface. The lower trailing CVP is denoted as surface trailing CVP in this paper, and for brevity we also call it trailing CVP (TCVP). This TCVP is usually thought to be due to the low-pressure recirculation zone and the suction effect of the major CVP. Figure 11 shows that TCVP rotates in the opposite direction to the major CVP and disappears as the jet plume lifts up downstream, which is an apparent feature for the farfield. The interesting part lies in the correlation of separation bubble with TCVP. Separation lines of $u / U_{\infty}=0.0$ is shown in figure 11(a)-(b) and there is no separation zone in figure 11(c)-(d). The enlarged view of the window in figure 11(a) clearly demonstrates that TCVP mainly locates in the separation bubble, or in the recirculation zone based on the separation bubble. This phenomenon clearly exists for both $J=5.5$ and $J=2.3$ case. In figure 11(b) the separation bubble is pushed aside from the centerline and its size decreases to be very small. Combing with figure 10, it is found that the separation bubble has a three dimensional configuration. In the jet leeward, the separation bubble has a large size especially in height. In the separation wing of the herringbone bubble, though the separation covers a large area, the height of the separation bubble is small and the whole separation region is attached to wall surface. The TCVP in Figure 11(b) is very clear to occur in domain with $u / U_{\infty}>0.0$, which is obviously kept outside of the separation bubble and it is quite different from the TCVP shown in figure 11(a). This is very interesting since no researchers mentioned this before. Literatures about jet in supersonic crossflow usually gave fuzzy description about TCVP in the jet leeward and led to an impression that TCVP is a single character both in the nearfield and farfield due to the suction of the major CVP. A careful observation exhibits that in the separation bubble of $J=2.3$ case in figure $11(\mathrm{~b})$, there is still a recirculation zone around small separation bubble. And it is clear that TCVP near to centerline is not in the recirculation zone but kept some distance from it. In figure 11(c) separation bubble vanishes and TCVR is totally located in favorable flow region. In figure 11(d) TCVP disappears completely in the farfield. Figure 12 gives the streamwise vorticity contours at different $\mathrm{x}$-locations. It is seen that the magnitude of vorticity of the TCVP is comparable to the major CVP in the jet vicinity and even larger than the major CVP at $x / D=8$. The vorticity of surface TCVP in figure $12(\mathrm{a})$ and (b) has the same sign while their configurations are varied. Thus very interesting questions are presented here, such as, whether the TCVP shown in figure 11(a) and 12(a) is occurring in the same vortex tubes with the TCVP shown in figure 11(b) and 12(b), since the two TCVPs shown in figure 11(a) and (b) or figure 12(a) and (b) are located in the separated and reattached regions, respectively. Since figure 11 and 12 only demonstrates the $2 \mathrm{D}$ flow character on different cross-flow slices in the flowfield, it is difficult to answer this question before detailed 3D flow topology is unveiled.

From figures $9-11$ it is seen that flowfields of $J=2.3$ and $J=5.5$ are similar. Since $J=5.5$ provides stronger jet interaction, we will mainly analyze the $J=5.5$ flowfield here after. Firstly, velocity profiles at the same wall-normal distance are shown in figure 13 for different streamwise locations. The velocity profiles have a $\mathrm{W}$-shape (the arrow points) corresponding to the herringbone separation. It is seen that in figure $13(\mathrm{a})$-(b) at $x / D=3$ and 6 , the velocity downstream of the jet is negative (dotted line in figure 13 represents $u / U_{\infty}=0.0$ ), which corresponds to a recirculation. Size of recirculation region approaches nearly zero at $y / D=0.4$, as shown in figure $13(\mathrm{c})$. Along the streamwise direction on $y / D=0.08$ plane, the velocity increases and at $x / D=9$ a positive velocity field is observed. At $x / D=14$, it is seen that the velocity profile has basically recovered and an earlier recovery occurs in in the reattachment region near to symmetric plane. At other wallnormal locations $(y / D=0.4$ and $y / D=0.6)$, this phenomenon is also apparent. The $\mathrm{W}$ shape width in velocity profile along wall normal direction converges significantly from 

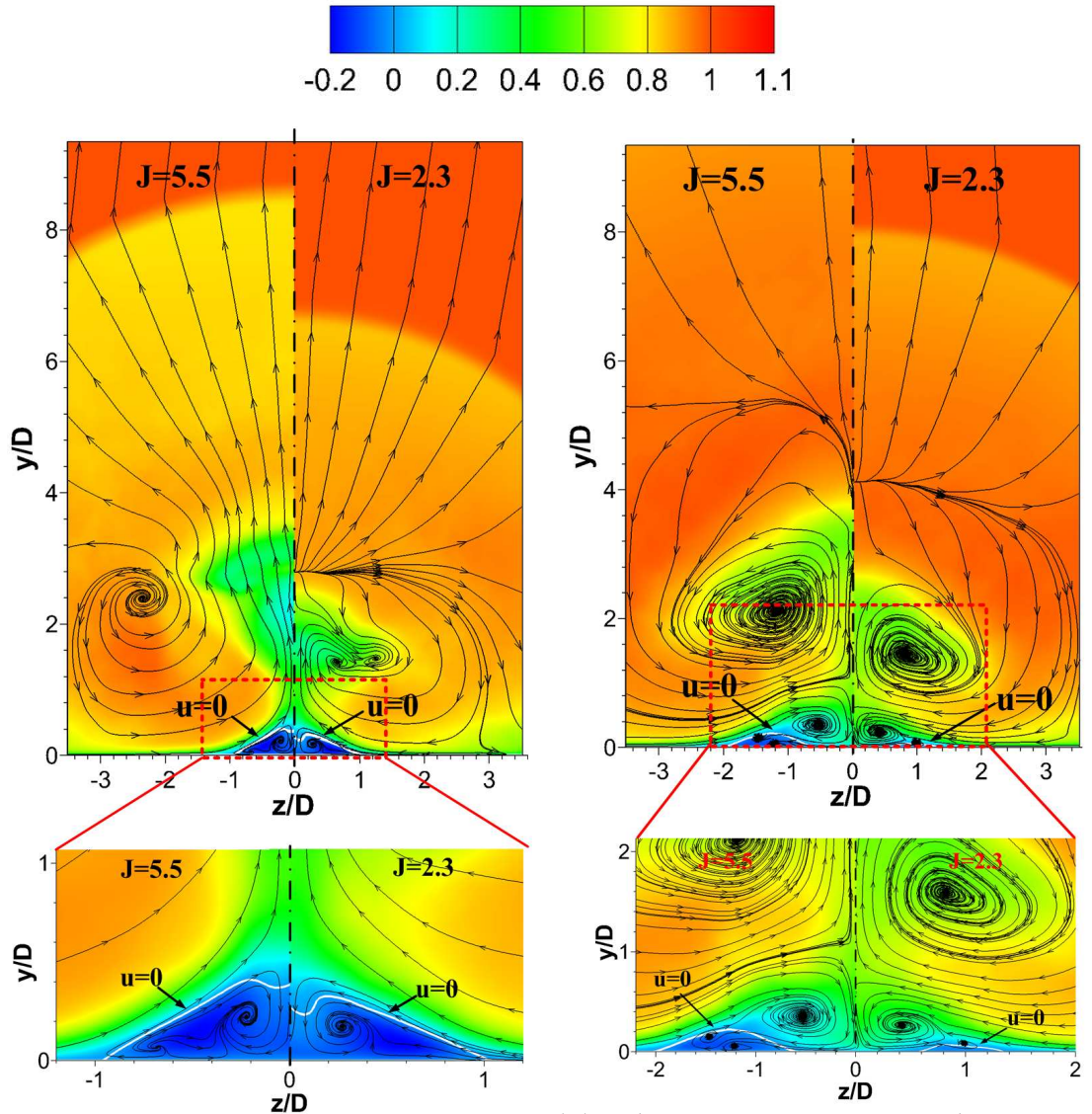

(a) $x / D=3.5$ for $J=5.5$ and $x / D=3.5$ for (b) $x / D=8$ for $J=5.5$ and $x / D=5.5$ for $J=2.3$

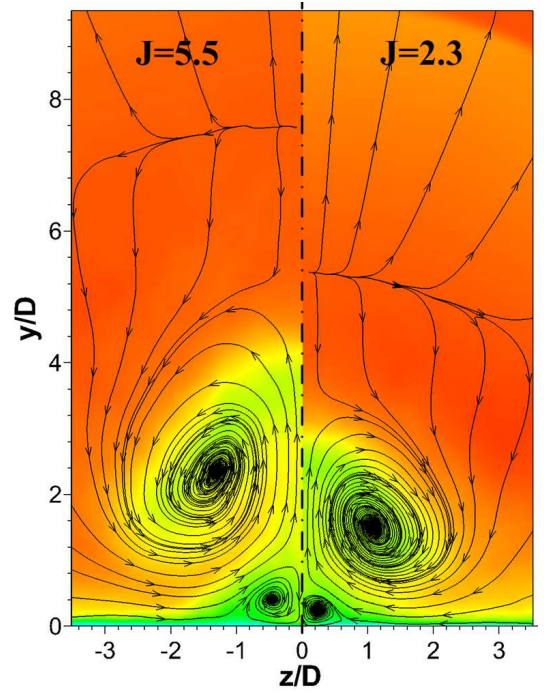
$J=2.3$

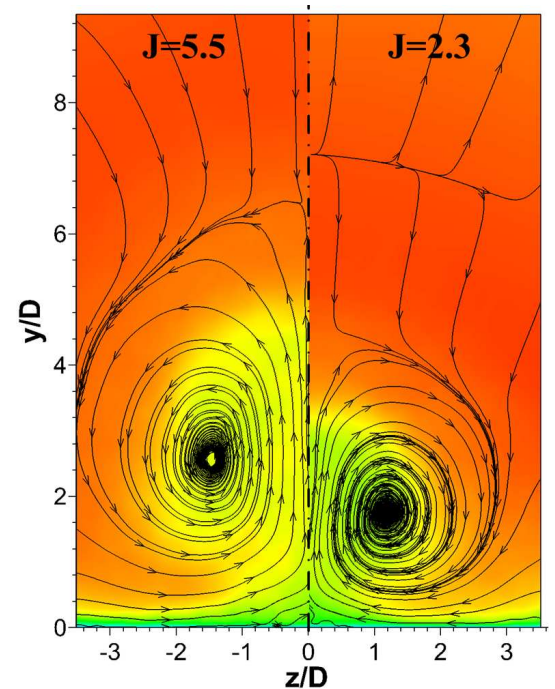

(c) $x / D=12$ for $J=5.5$ and $x / D=8$ for (d) $x / D=18$ for $J=5.5$ and $x / D=12$ for $J=2.3$ $J=2.3$

Figure 11. Contours of the mean flow streamwise velocity and streamlines on cross planes of $J=5.5$ and $J=2.3$, isolines of $u / U_{\infty}=0.0$ are superimposed 

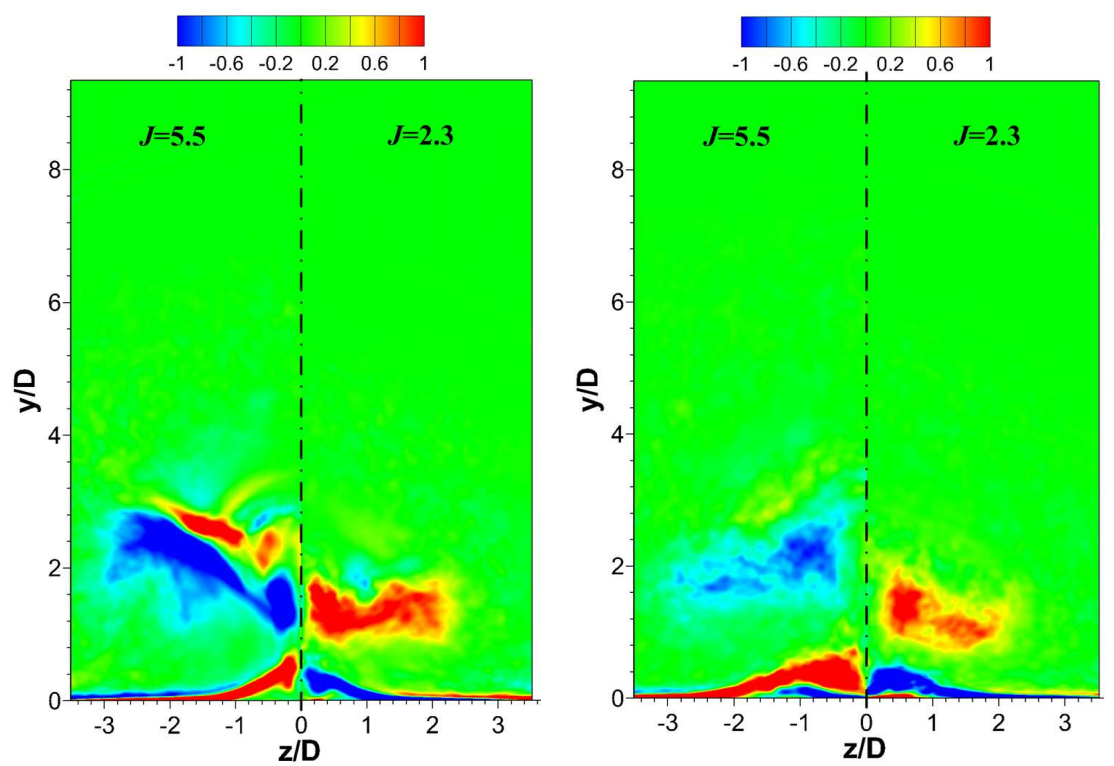

(a) $x / D=3.5$ for $J=5.5$ and $x / D=3.5$ for (b) $x / D=8$ for $J=5.5$ and $x / D=5.5$ for $J=2.3$

$J=2.3$

FIGURE 12. Contours of the time averaged streamwise vorticity on cross planes of $J=5.5$ and $J=2.3$.

$y / D=0.08$ to $y / D=0.6$, which means the region affected by the jet is decreased in $y$-axis. On $y / D=0.6$ plane, the velocity profile at $x / D=6$ is completely positive, which indicates the recirculation is located under $y / D=0.6$ plane. Separation width from the velocity profiles also converges from $y / D=0.08$ to $y=0.2$ plane, which reflects that the separation is likely to be a bump on the surface.

Several conclusions could be made from the above analysis. The first is that TCVP shown in figure 11(a) and 11(b)-(c) is located in totally different velocity regimes. The TCVP in figure 11(a) is in a separation region while the TCVP in figure 11(b)-(c) is in a zone with positive velocity. From this point, these TCVPs are inevitably different in formation. It is appropriate to denote the TCVP shown in figure 11(a) as the primary TCVP and that in figure 11(b) as a secondary TCVP. The second is that the separation region has a complex $3 \mathrm{D}$ configuration, the edge of the separation region is located below $y / D=0.6$ and the separation bubble has a decreased width along $y$-axis.

Time-averaged pressure and Mach number contours at various locations along with streamlines are shown in closer-view in figure 14, which are consistent with the analysis on figure 13. Separation line of $u / U_{\infty}=-0.0001$ is shown in figure 14 (a) with white solid line. It is seen that the herringbone recirculation has two wings, which corresponds to two oblique symmetric separation regions. Between the wings there is a zone with positive velocity, which means that a reattachment occurs and it is embayed by the separated zone, as shown in the dotted-line window. A reattached point with $u / U_{\infty}=-0.0001$ in the centerline is also marked in figure 14(a). The streamlines clearly show the inner flow structure of recirculation zones. The pressure contour shown in figure 14(a) demonstrates that a low pressure exists in jet leeward due to the blockage and expansion effects from the jet. Crossflow runs around the jet and impinges together in the jet leeward, which leads to a local pressure increment due to the collision effects. Remember that this collision shock corresponds to the secondary shock in Figure 1(a). It is identified that 


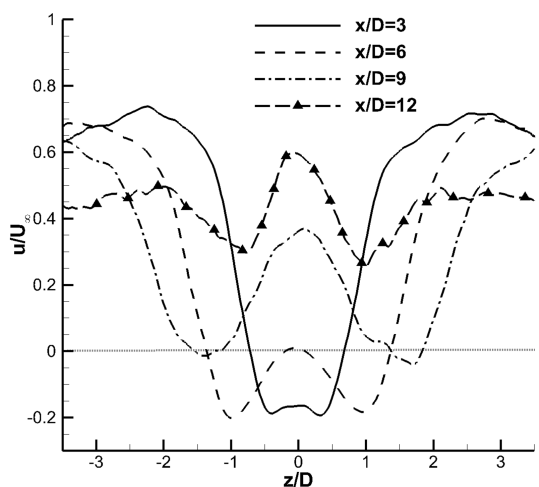

(a) $y / D=0.08$

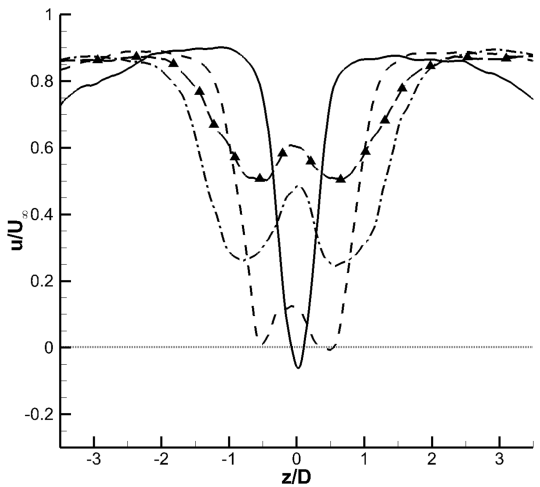

(c) $y / D=0.4$

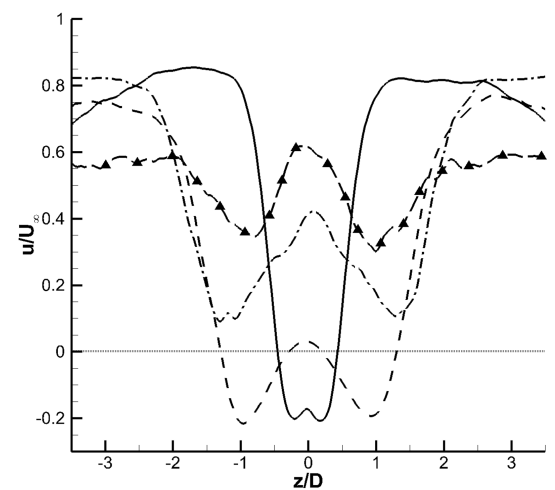

(b) $y / D=0.2$

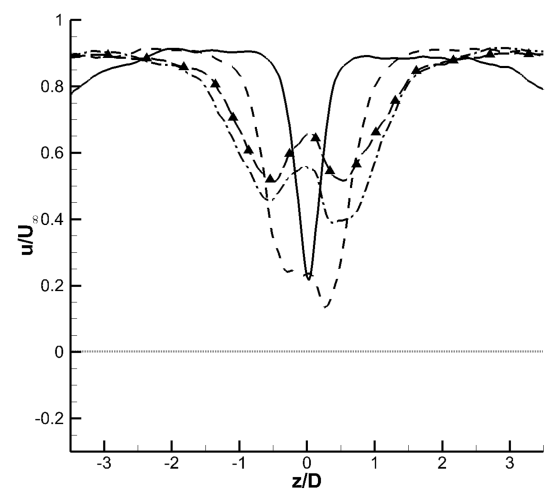

(d) $y / D=0.6$

FiguRE 13. Time-averaged velocity profile at different wall normal location of $J=5.5$ case, dotted line represents $u / U_{\infty}=0.0$

pressure after the separation line drawn in figure 14(a) increases which implies existence of a shock/boundary layer interaction. From figure 14(b) we can see that crossflow near the wall in the jet lateral is supersonic. This is expected since the separation region ahead of the jet pushes crossflow high away from surface and a sudden expansion after the separation bubble occurs in the negative $y$-axis. The crossflow running into the jet leeward experiences further expansion in the spanwise direction. These two expansions cause that flow speed becomes supersonic and it is sure that collision shock would have a foot standing in the recirculation. As shown in figures 14(a)-(c), when the wall-normal distance increases from $y / D=0.0$ to 0.5 , the separation region ends further upstream and this is due to the three-dimensionality of the separation bubble. At $y / D=0.5$, there is no obvious recirculating flow. The separation region in the jet wake and the reattachment region can also be identified in figure 14(d), which shows Mach number contours and a superimposed separation line.

In figure 14 it is confirmed again that the TCVP in figure 11(a) and 11(b) are different vortices since the velocity fields are completely different. The counter-rotating character of the primary TCVP is not only related to the recirculation in the spanwise direction, but also connected to the recirculating flow opposite the incoming flow.

From figures 14(a)-(b), it is seen that the herringbone region fades some distance behind the separation wing. It is expected since streamlines running around the jet impinge on the wingtip of the herringbone separation and result in a wing interception, which indicates that the interaction intensity of the major CVP reduces significantly at 
the wingtip location since the major CVP has been lifted to a higher position. Due to the impingement of the lateral supersonic flow around the jet, the trailing wingtip is converged. At the same time the reattachment flow extends in the reattachment zone (shown in the dashed-line window of figure 14a), impinges the separation bubble and blows off the near-wall separated flow. It is identified since in figure 14(a) streamlines in the reattachment region run into the separation wing region and the downstream zone in the farfield. The cross interaction in this wingtip region leads to the termination of the oblique trailing separation wings. Downstream of the vicinity of reattachment region, there is an expansion zone in which diverging streamline impinges with crossflow and forms a slip line, as shown in figure 14(a). This expansion zone is attached to wall since no such phenomenon is found in figure 14(c). The reattachment supersonic flow impinges on the wall and leads to a pressure increment in the downstream region of the herringbone separation bubble. As shown in figure 10(c), a weak reattachment shock is generated after the flow expansion over the separation wing.

Time-averaged Reynolds stress and TKE (turbulent kinetic energy) contours at various locations are shown in figure 15. The asymmetry in the spanwise direction in Reynolds stress and TKE distribution along the centerline $z / D=0.0$ is due to limited averaging time. At $y / D=0.4, \overline{u^{\prime} v^{\prime}}$ and TKE with high intensity occurs in the collision shock (as seen in figure 15c) region and fades in the reattachment region. Streaky structures with TKE and negative $\overline{u^{\prime} v^{\prime}}$ in a high magnitude re-occurs in the downstream, which represents the formation of fresh turbulence. Recalling figures 11-12, the secondary TCVP are likely to be the main reason. In the farfield at $10.0<x / D<15.0$, the levels of $\overline{u^{\prime} v^{\prime}}$ and TKE in the wake flow are gradually smeared and the intensity approaches uniform downstream of $x / D=15.0$. This phenomenon indicates the vanishing of the local coherent structures. $\overline{u^{\prime} v^{\prime}}$ and TKE profiles at $y / D=0.4$ are shown in figure 16 . It is seen that in the vicinity of $z / D=0.0$, the $\overline{u^{\prime} v^{\prime}}$ and TKE profiles at $x / D=8.0$ have an obvious augment in magnitude. At $x / D=12$, it is seen that the $\overline{u^{\prime} v^{\prime}}$ and TKE profile has basically recovered in the reattachment region near to symmetric plane $z / D=0.0$. This corresponds to the contours shown in figure $15(\mathrm{a})-(\mathrm{b})$.

Iso-surfaces of the streamwise velocity of the $J=5.5$ and $J=2.3$ cases are shown in figure 17 to demonstrate the 3D near-wall wake structures, colored by the local wallnormal distance. The 3D separation bubble looks like a herringbone hill. The jet leeward separation bubble is a whole region and further downstream is spilt by a reattached flow to two separate separation wings. The peak of the oblique wing corresponds to the edge of the separation zone. The $\mathrm{V}$-shape valley between the separation wings corresponds to the reattachment region, which has an obvious $3 \mathrm{D}$ structure. It is expected that the flow experiences an expansion when going over the top of the separation hills and falls into the $\mathrm{V}$-shape reattachment valley. Combined with the analysis of $2 \mathrm{D}$ streamlines on $y / D=0.2$ plane, as shown in figure $14(\mathrm{~b})$, it is indicated that the reattached flow directly supports the secondary TCVP downstream of the reattachment valley, which is different from the primary TCVP. Results for the $J=2.3$ case are shown in figure $17(\mathrm{~b})$ and it is seen that lower $J$ case has a similar character of flow configuration, though the size is smaller than the $J=5.5$ case. A careful observation shows that the size of jet leeward separation of $J=2.3$ case is almost kept unchanged comparing to $J=5.5$ case, while the separation wing is much shorter. This phenomenon further confirms that the collision shock is independent upon $J$, which leads to a leeward separation in similar size.

The schematic of the recirculation zone structures in jet wake flow is illustrated in figure 18. The bow shock ahead of the jet, the horseshoe vortex that wraps around the base of the jet and the major CVP are well known, thus they are omitted and will not be discussed in detail in this paper. Figure 18(a) shows a classic flow topology of an oblique shock 


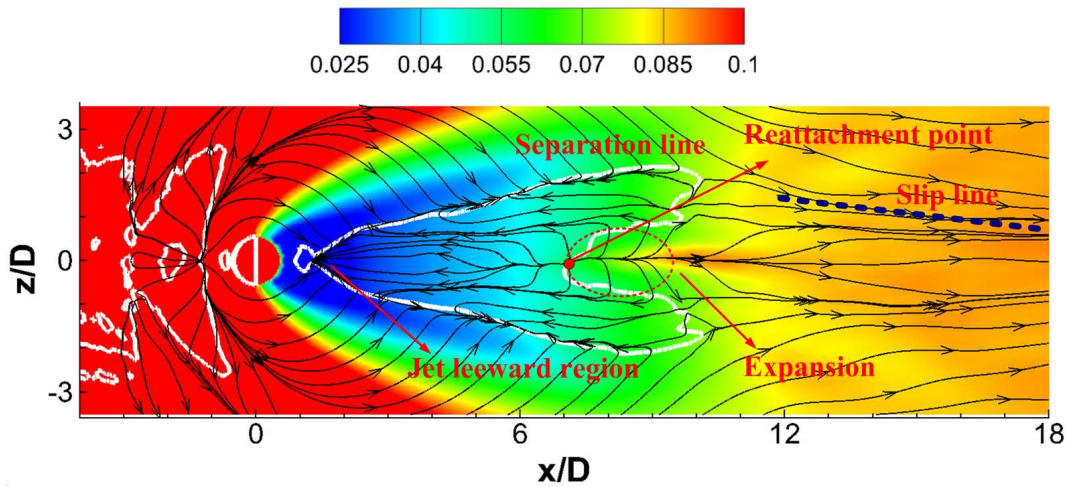

(a) pressure contour with streamlines at $y / D=0.0$

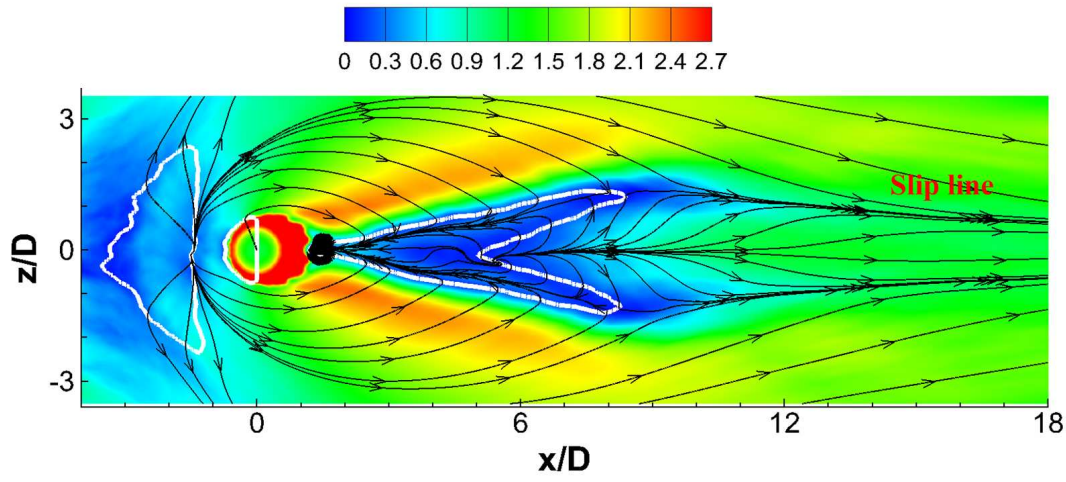

(b) Mach number contour with streamlines at $y / D=0.2$

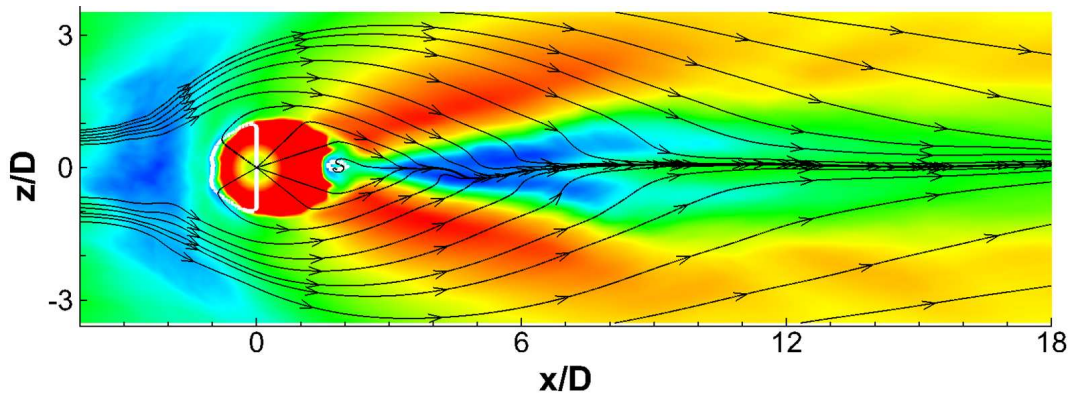

(c) Mach number contour with streamlines at $y / D=0.5$

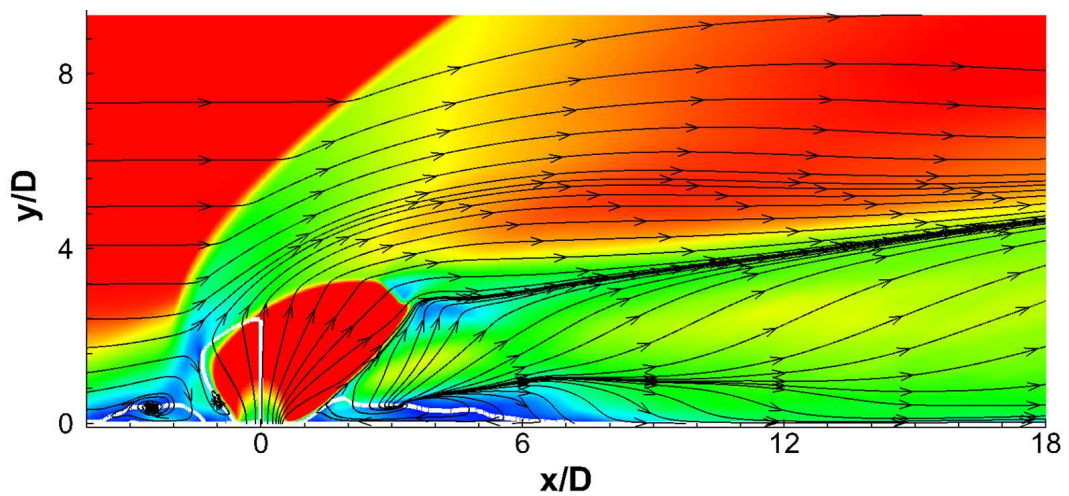

(d) Mach number contour with streamlines at mid-span plane $z / D=0.0$

Figure 14. Contours of time-averaged pressure and Mach number contours and streamlines on different planes of $J=5.5$. Separation line $u / U_{\infty}=0.0$ is superimposed 


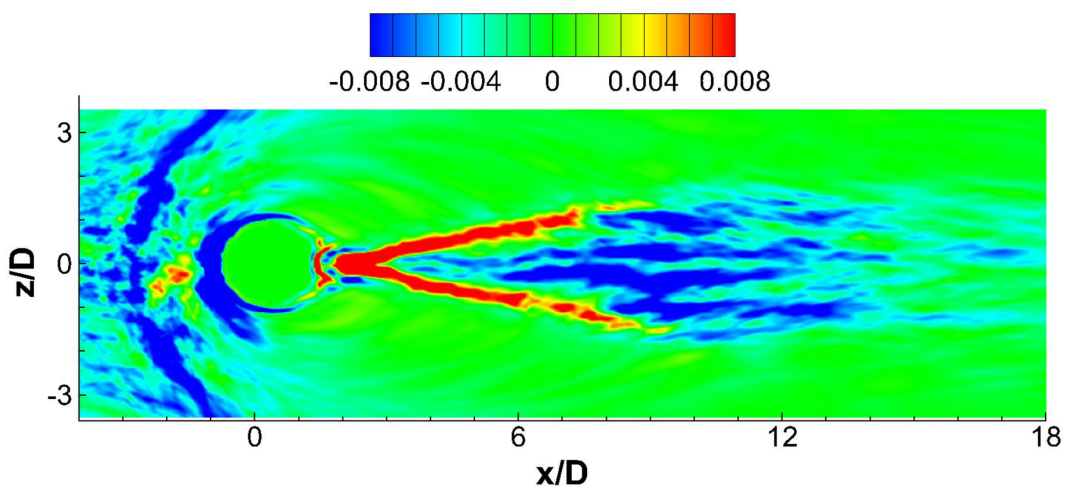

(a) contour of $\overline{u^{\prime} v^{\prime}}$ at $y / D=0.4$

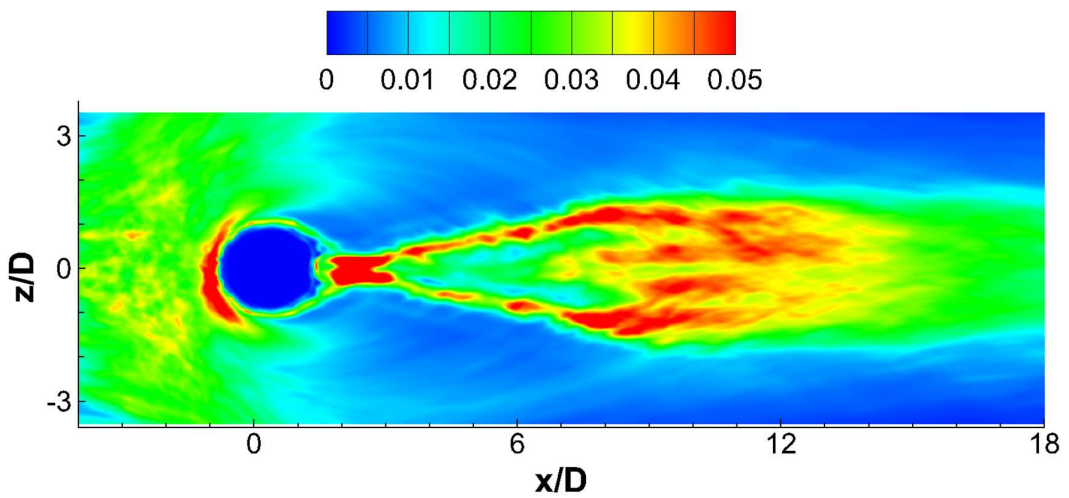

(b) contour of TKE at $y / D=0.4$

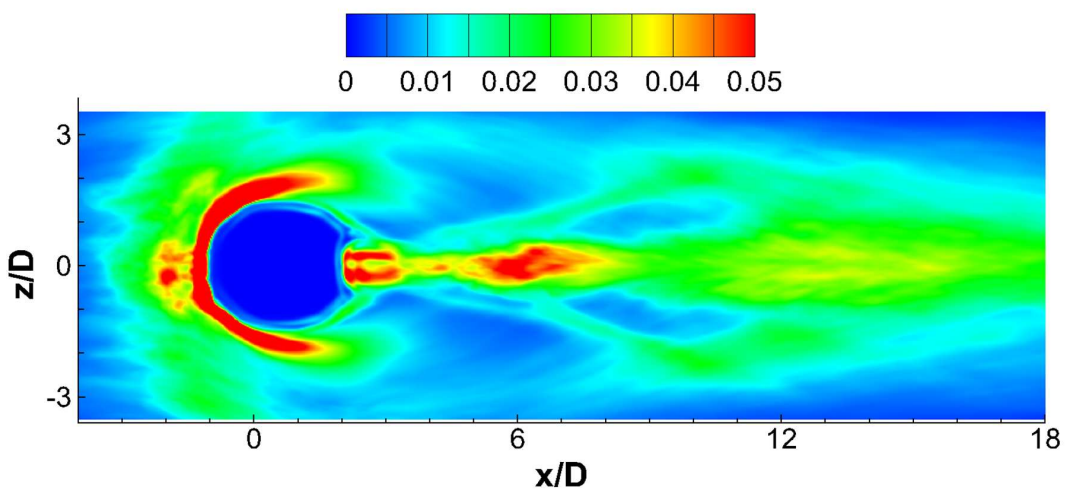

(c) contour of TKE at $y / D=1.0$

FiguRE 15. Contours of the time averaged Reynolds stress and TKE on different horizontal planes of $J=5.5$. non-dimensionalized by $U_{\infty}^{2}$

wave interaction with a supersonic boundary layer, which demonstrates the recirculating flow has an opposite direction to the incoming flow. After the separation bubble peak, expansion occurs and is followed by a reattachment shock. The reattachment shock in figure 10(c)-(d) and pressure increment in figure 14(a) can be explained by this schematic. Figure 18(b) demonstrates the complex configuration of the collision shock interaction with the lateral supersonic boundary layer. The shock wave surface originates from the collision of the supersonic flow running around the jet barrel, as also shown in figure 1(a) 


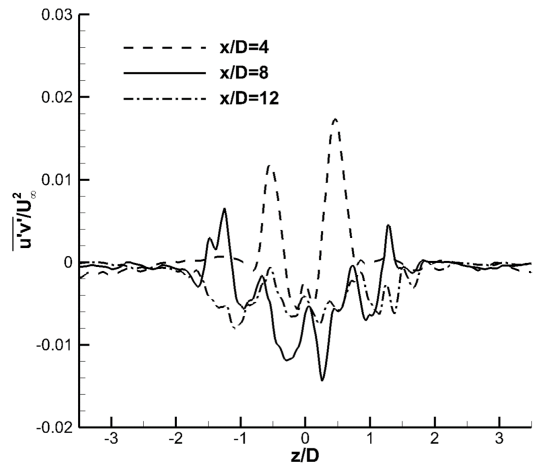

(a) Reynolds stress $\overline{u^{\prime} v^{\prime}} / U_{\infty}^{2}$

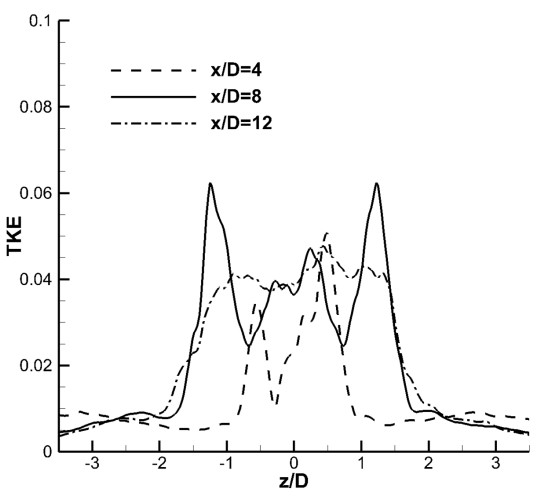

(b) TKE

Figure 16. Time-averaged Reynolds stress and TKE profiles at $y / D=0.4$ of $J=5.5$ case, non-dimensionalized by $U^{2} \infty$.
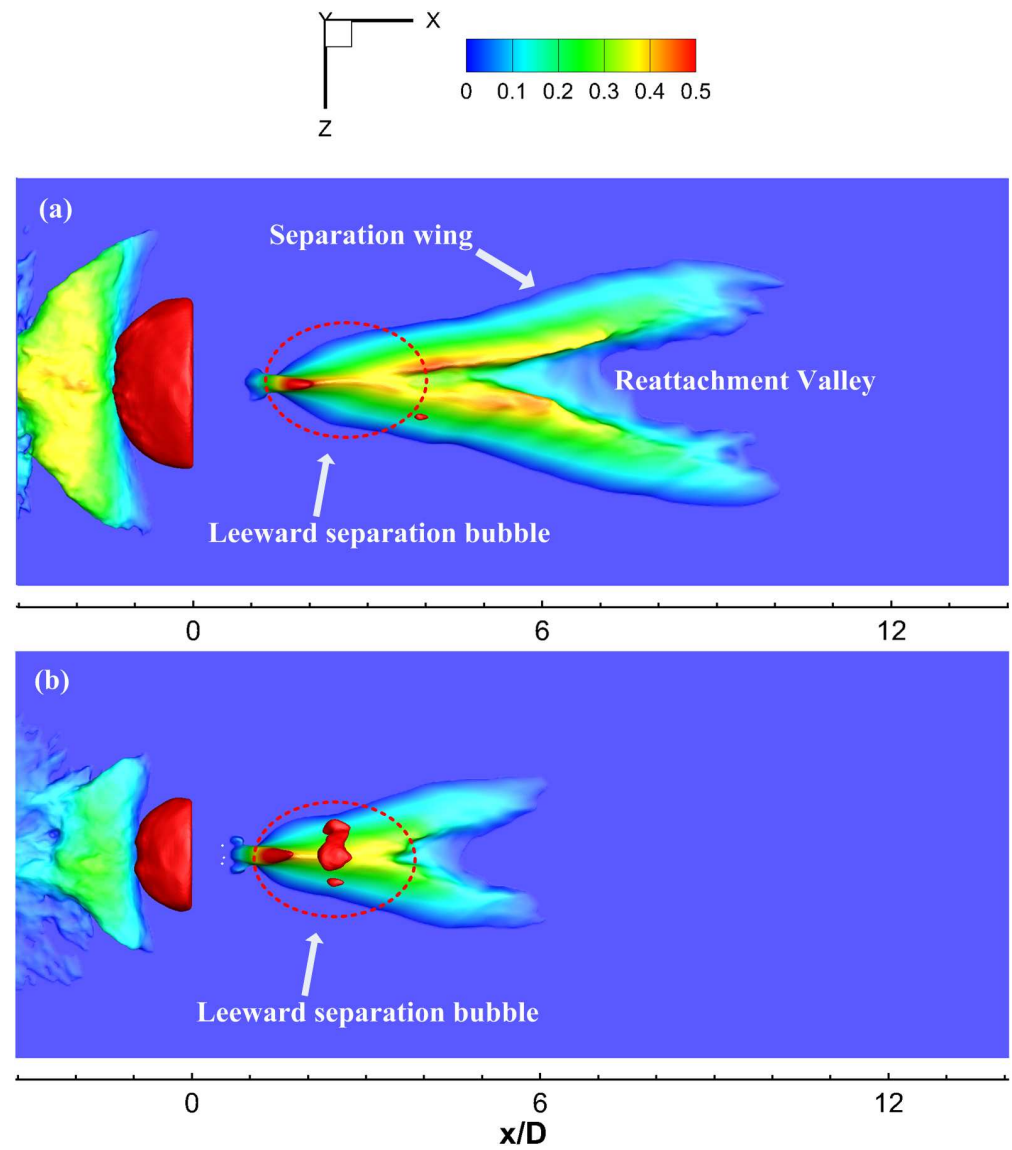

FiguRE 17. Iso-surface of streamwise velocity $\left(u / U_{\infty}=0.0\right)$ colored by local wall-normal distance $(y / D)$ of (a) $J=5.5$ and (b) $J=2.3$ cases 
from previous literatures. It should be mentioned that since collision shocks have been twisted by the induced separation, shock structures could not be observed as regularly as described in Figure 18(b). The collision shock surface is located under the major CVP and oblique shock surfaces are generated along the streamwise direction vertically to the wall. Since the separation bubble is induced in the jet leeward, the flow behind collision is also blocked by the separation bubble and a shock surface is formed in the wall-normal direction. These shock surfaces merge and form a 3D unified half-cone-like shock surface, as shown in red color in figure 18(b).

As shown in figure 18(b), the induced herringbone recirculation extends in the spanwise direction like a foot of the incident collision shock into the incoming lateral supersonic flow. Behind the oblique separation wing, the reattachment domain colored in grey in the sketch, could be deduced to exist in the V-shape valley. It is predicted that at least three streams come into the reattachment valley. Two streams are from the flow running around the jet lateral and across the oblique separation wing, and the other one is from flow going through jet leeward and across the reattachment point. In next section 3D streamlines will be given to demonstrate these flow patterns. Figure 18(b) shows the separation and demonstrates the TCVP on slice cuts in the recirculating flowfield, which is consistent with the primary TCVP assumption. This means that the primary TCVP occurs in the separation bubble to rotate and recirculate. The secondary TCVP cannot be explained by using figure 18(b). In next section we will use 3D streamlines to analyze the flow topology, especially to discuss the secondary TCVP.

\subsection{Flow topology analysis of surface TCVPs}

In this section we will show 3D time-averaged streamlines running into jet wake region and analyze their flow patterns. The key purpose is to unveil the mechanism of formation of surface TCVPs. Figure 19 gives representative streamlines which originate from an oblique line parallel to separation wing on the iso-surface of $u / U_{\infty}=0.05$ in the jet lateral. These streamlines reflect flow motions in the jet wakes. It is found that different types of flow patterns form in the wake. Some streamlines run around the jet barrel and concentrate in the jet leeward and rise up into farfield. Some streamlines run across the leeward separation bubble, recirculate and converge at the jet leeward. Others run across the separation wing, rotate and move into downstream region. It is necessary to understand clearly flow motion in the jet wakes, especially the flow topologies corresponding to surface TCVPs.

Figure 20 demonstrates different types of detailed flow patterns separately for clarity. Streamlines are generated in the same way as figure 19. Figure 20(a) shows the streamlines originating from the supersonic crossflow converging in the jet lee and going directly downstream, which is denoted as Type I pattern. These streamlines rise up in the jet lee following the suction of the major CVP and run into the core of the jet plume in the farfield. Analysis shows that these streamlines mainly originate from the supersonic crossflow in the vicinity of the symmetric plane. Together with figures 14(a)-(b), it is concluded that the streamlines in the jet lateral region pass the expanded supersonic zone and collide with another supersonic stream from the opposite side, which converges the flow and generates $\mathrm{V}$-shape collision shock waves. This collision also leads to the generation of the jet leeward separation and further induces the herringbone trailing separation in the boundary layer, as described in figure 18(b).

Figure 20(b) demonstrates the Type II flow pattern with transparent iso-surface of $u / U_{\infty}=0.05$ to reveal the flow in the interior of the separation bubble. For this pattern, the streamlines go across the jet leeward separation bubble and cluster in the reattachment valley, and then recirculate following the leeward separation bubble which acts like 


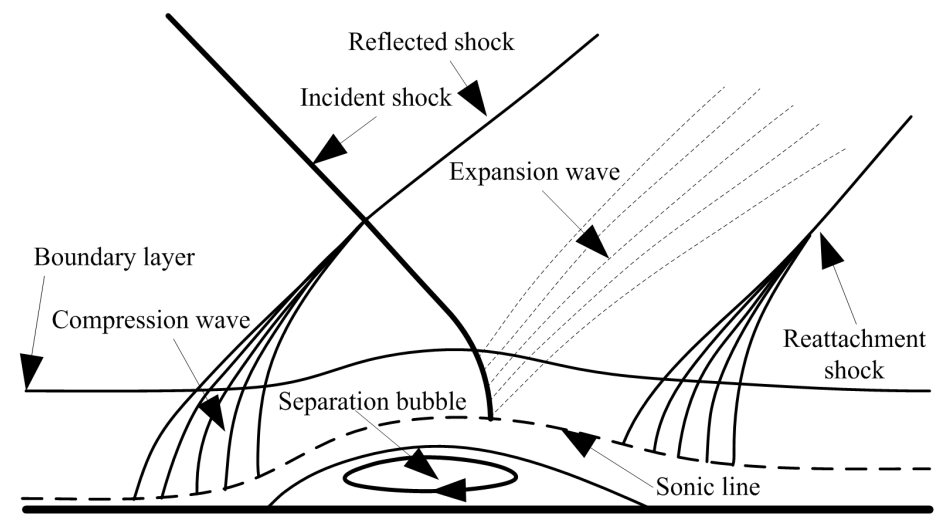

(a) classic schematic of an incident oblique shock interacting with a supersonic boundary layer, modified from Ref (Gaitonde 2015)

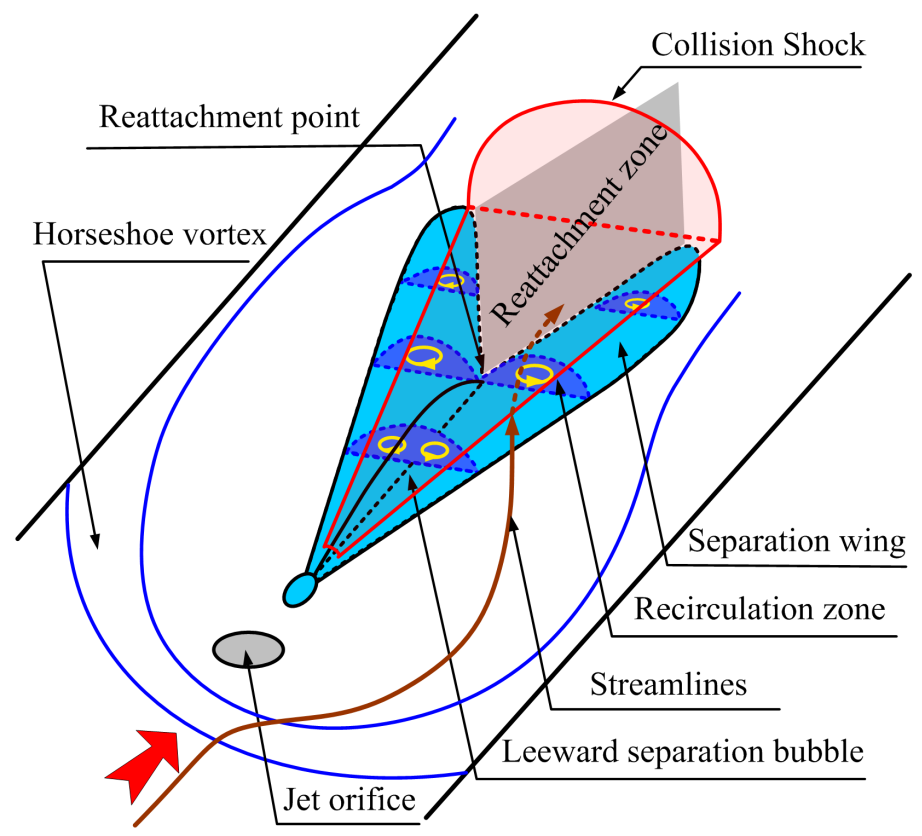

(b) separation induced by collision shocks and primary TCVP on a slice cut from the herringbone recirculation zone

FiguRE 18. Schematic of herringbone oblique recirculation zones induced by collision shock waves behind the transverse jet injected into a supersonic crossflow

a tunnel to let streamlines penetrate. The penetration procedure is very clear with streamlines colored by local wall normal distance. Combining with figure 14(a), it is inferred that streamlines of Type II in the vicinity of the reattachment point come into the recirculation and move against the freestream direction. This recirculating flow along the oblique separation tunnel is just what has been described as the primary TCVP in Section 3.2. The streamlines concentrate in the jet leeward and rise up due to the major CVP. Type II streamlines rotate when penetrating the recirculation zone. This is due to the rotation character of the recirculating flow in the spanwise direction, which has been well described in figure 18(a). 


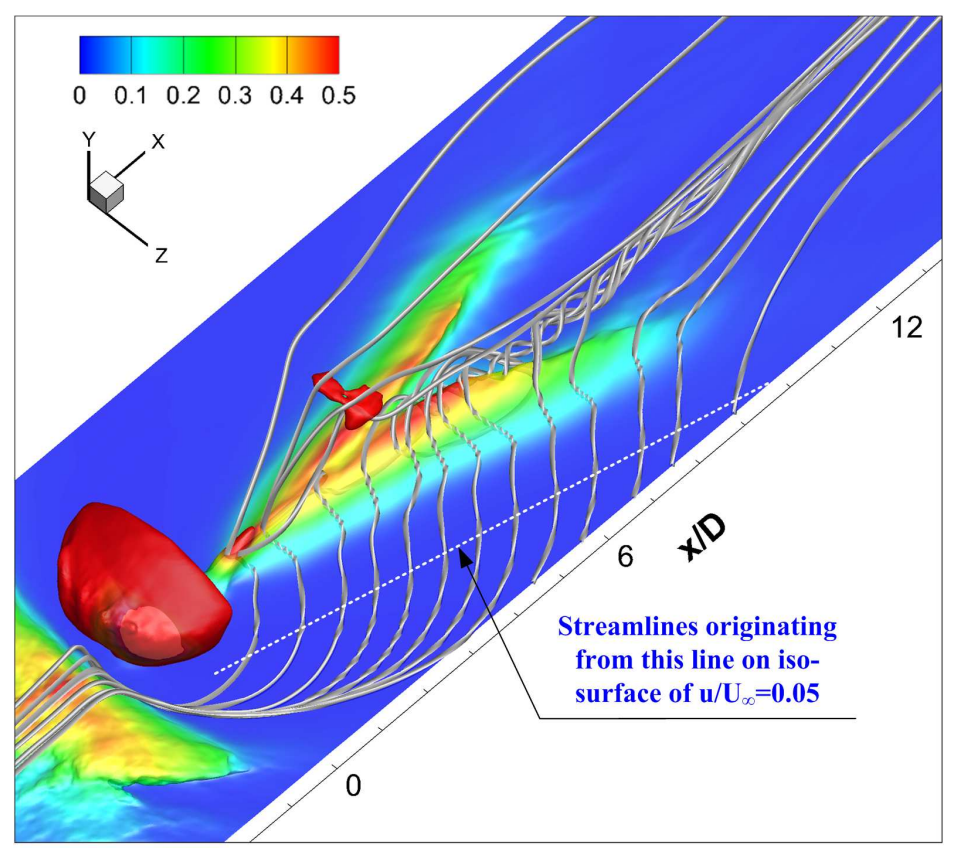

FiguRE 19. Flow topology demonstrated by $3 \mathrm{D}$ streamlines in the jet wake of $J=5.5$, with iso-surface of streamwise velocity $\left(u / U_{\infty}=0.05\right)$ colored by local wall-normal distance

Figure 20(c) demonstrates the Type III flow pattern. This pattern is believed to well explain the secondary surface TCVP shown in figure 11(b)-(c). For type III, the jet lateral flow is supersonic ahead of separation line and the herringbone separation wing acts as a micro-ramp vortex generator. Those streamlines go over the separation-wing bubble or separation hill and rotate to form a swirling flow and roll into a concentrated vortex rope, to form the secondary TCVP. This procedure only can be displayed in a 3D wake configuration with 3D streamlines. From the analysis given in Section 3.2, it is known that secondary TCVP is located in the reattachment valley with a positive velocity. This flow pattern is definitely different from Type II streamlines, which mainly locates in separation zone with a negative streamwise velocity. In the separation wing, there is a combined flow pattern which has the partial character of Types II and III, denoted as Type IV. For this pattern, streamlines go over the wing, run into the reattachment valley and recirculate through the separation wing interior downstream of the reattachment point. Further these streamlines penetrate out of the separation wing and follows the secondary surface TCVP to move downstream. Type IV has the recirculating character of Type II and rotating character of Type III. A comparison between figure 16 and figure 10 indicates that the primary TCVP shown in figure 11(a) mainly corresponds to the Type II flow pattern. For figure 11(b), existence of the separation wing on slices indicates a combined pattern of Type III and IV. For figure 11(c), there is no separation and the secondary TCVP comes from the combined effects of Type III and IV.

The main difference between Type III and Type II is that Type III streamlines go over the separation bubble and directly run into the reattachment valley, which means the streamlines mainly move outside of the separation region. Streamlines of Type II penetrate in leeward separation bubble and rotate following the swirling vortex in the recirculation region. Streamlines in Type III and IV rotate in the reattachments valley outside of the separation and further lose the swirling strength downstream of the 
reattachment valley due to the disappearance of herringbone separation and the local dissipation, which corresponds to the terminating of secondary surface TCVP. Decay of TCVP strength in the farfield can also be deduced from vortex dynamics since the two counter rotating vortex in TCVP induces each other and they lift off the surface as a result of upwash.

The main features of the micro-ramp wake region are presented in figure 21 to demonstrate the formation of the secondary TCVP. Babinsky et al. (2009) presented a schematic (figure 21a) to show that a primary pair of counter-rotating vortices rolls up gradually and is strengthened on the two sides of the micro-ramp, which finally converge near the trailing edge of the ramp. Wang et al. (2015a) traced the streamlines and found that streamlines in the inner boundary layer were engulfed into the primary vortex core (shown in Figure 21b) and streamlines in the outer layer simply pass over the ramp. The jet lateral flow shown in figure 20(c) near the wall passes the separation-wing bubble, where an upwind ramp plays the same role as a micro-ramp in the boundary layer. The formation of the primary vortex in micro-ramp flow is the same as the secondary surface TCVP in jet wake flow. Many literatures have discussed the mechanism of micro-ramp flow, detailed analysis is omitted here for brevity.

It is inferred that local pressure difference plays a dominant role in the flow recirculation or rotation. Figure 22 demonstrates the pressure contour on the iso-surface of $u / U_{\infty}=0.0$ with representative streamlines of Type II, III and IV. In the jet lee, a bow low pressure region exists due to the blockage and expansion effects from the jet barrel. The pressure recovers at the reattachment valley. Together with figure 14(a), it is indicated that streamlines from the jet lateral region run into the reattachment valley would increase the local pressure to a higher level over the surrounding area. The high pressure flow in the reattachment region pushes the streamlines into the herringbone recirculation region. Type II streamlines go across the separation bubble and are pushed into the jet leeward recirculation zone. The streamlines recirculate and rotate into the jet lee. Type IV streamlines are also pushed by the high pressure of the reattachment region into the oblique separation wing. Streamlines of Type III go over the separation wing and directly acquire the rotation momentum by the blockage of high pressure in the reattachment valley. These streamlines swirl downstream of the herringbone zone and would not roll into any recirculation region. Figure 23 gives the front view of these three streamlines. Compared to figure 11, Type II streamlines represent the rotation of the primary TCVP, while Type III streamlines represent the rotation of the secondary TCVP. The streamlines on slices in figure 11 are the projection of the $3 \mathrm{D}$ streamlines. Type II streamline rotates as well as recirculates in the jet leeward separation bubble. Type IV streamline recirculates in the separation wing firstly and then follows secondary TCVP to move. The analysis further validates the conclusion that primary TCVP and secondary TCVP are different patterns in the jet wake flow.

Figure 24 shows cluster of streamlines which originate from an oblique line parallel to the separation wing on the iso-surface of $u / U_{\infty}=0.05$ and 0.5 from jet lateral side. It is found that streamlines with different pattern are driven by flow and move into different region. Streamlines in figure 24(a) demonstrate the secondary TCVP structure and in figure 24(b) demonstrate the primary TCVP. It is interesting to see that on the wingtip of the separation bubble, a new shedding vortex is induced and streamlines rotate from the wingtip and the swirling strength fades some distance downstream. In figure 24(c)(d) with streamlines originating from iso-surface of $u / U_{\infty}=0.5$, both Type II and III flow pattern are found. The main difference of figure 24(c) from figure 24(a) is that the acquired rotation magnitude decays earlier, which indicates that the separation ramp mainly collects the low-momentum streamlines from the inner boundary layer, acting 


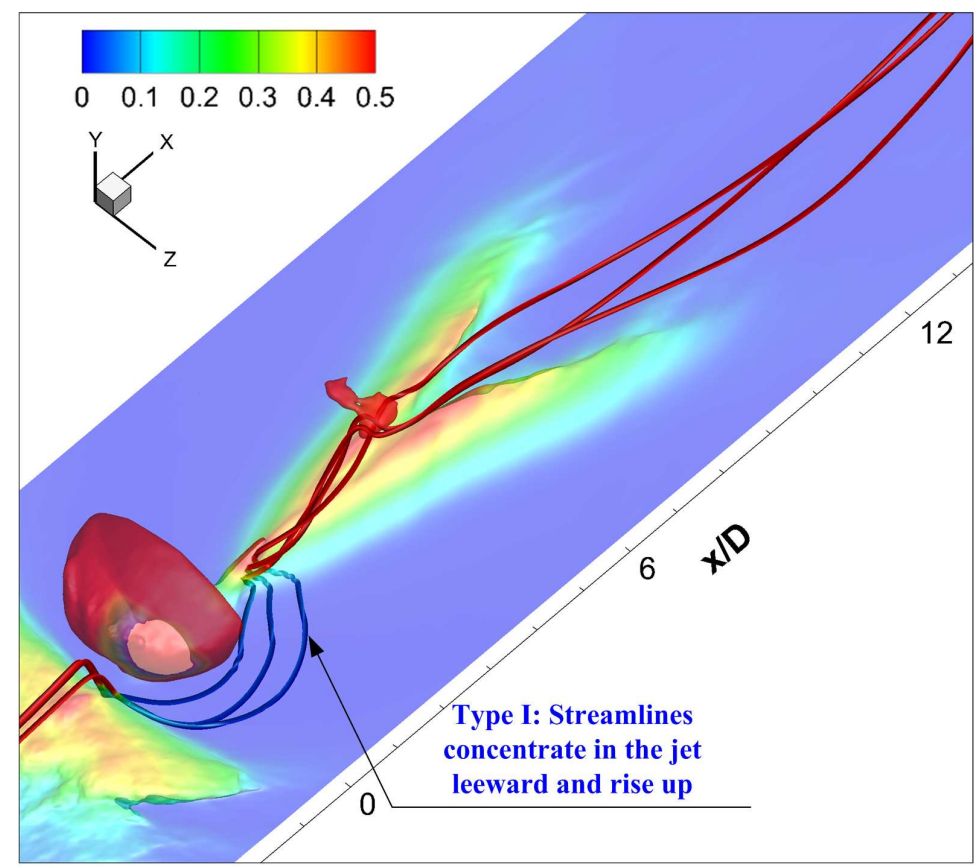

(a) Type I pattern, streamlines concentrate in the jet leeward and rise up following the suction of the CVP

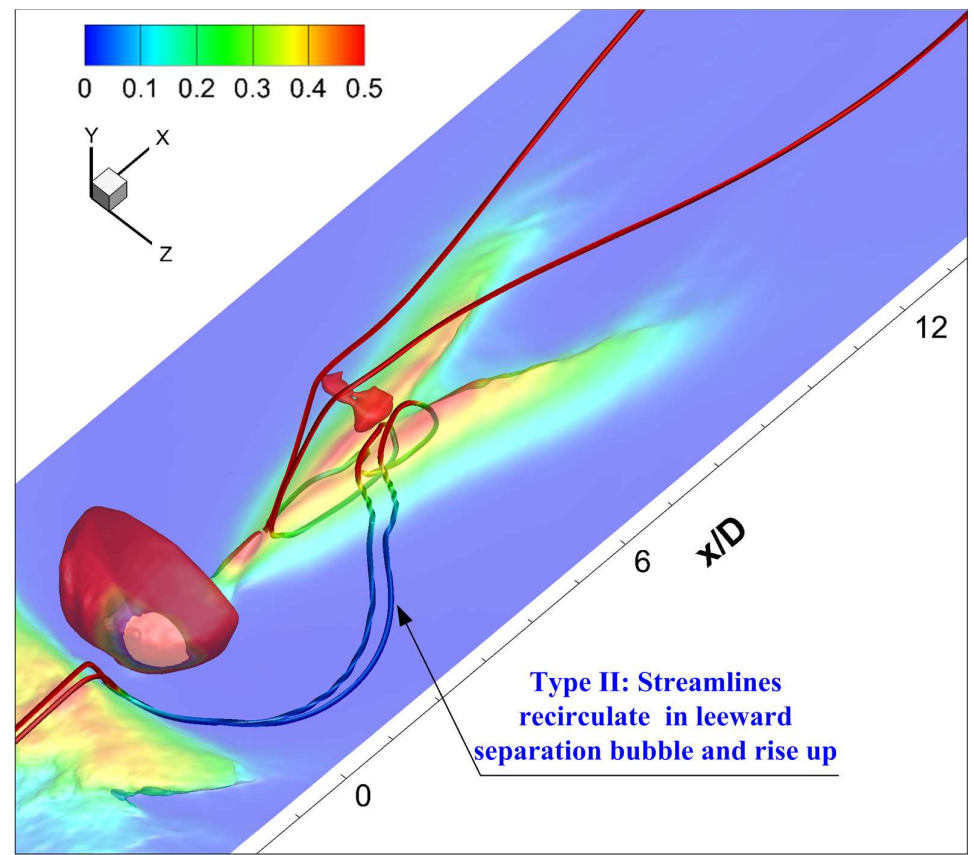

(b) Type II pattern, streamlines recirculate through leeward separation bubble, concentrate in the jet leeward and rise up 


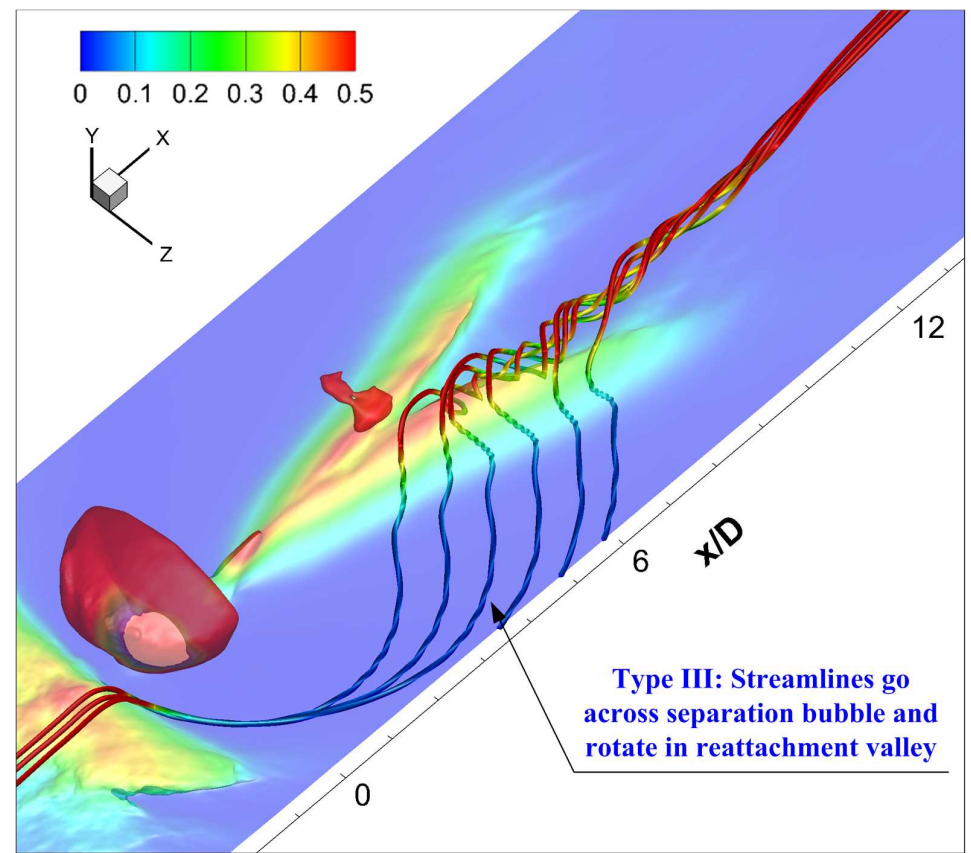

(c) Type III pattern, streamlines run across separation wing and roll to form a vortex rope

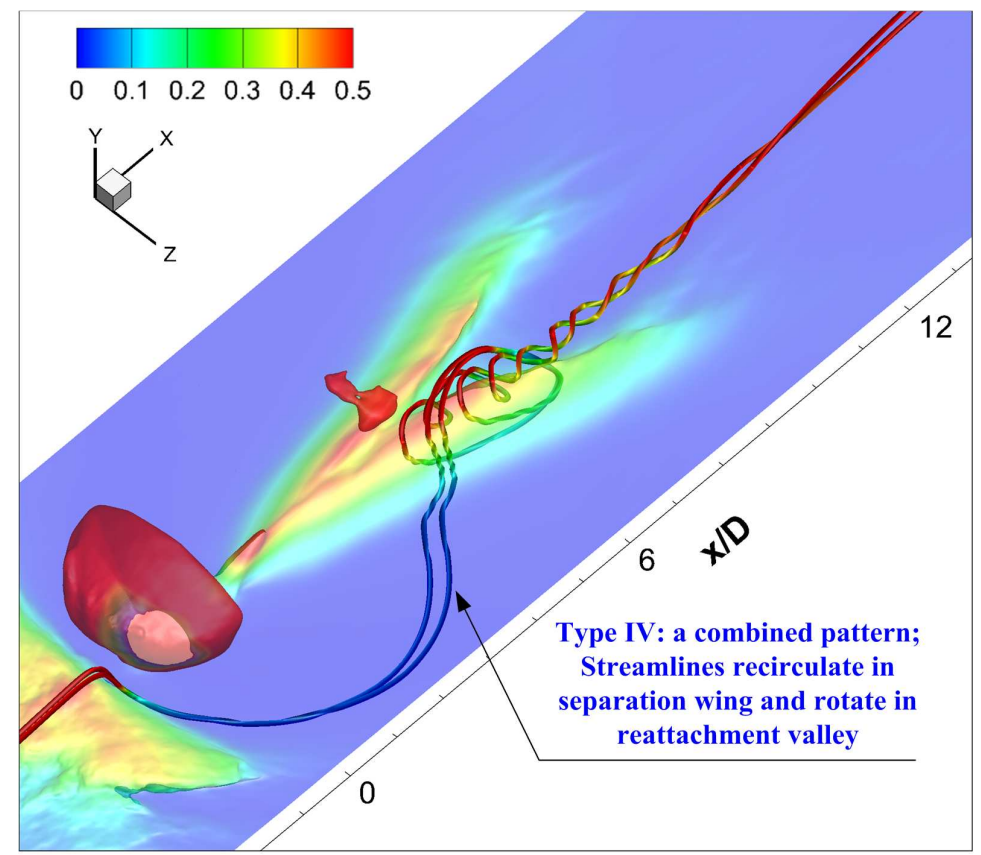

(d) Type IV pattern, streamlines run across separation wing and go through the separation zone and rotate into the vortex rope shown in type III

Figure 20. Flow patterns (Type I, II, III, IV) demonstrated by streamlines in jet $(J=5.5)$ wake to demonstrate different types of trailing vortices, with transparent iso-surface of $u / U_{\infty}=0.05$, colored by local wall-normal distance 


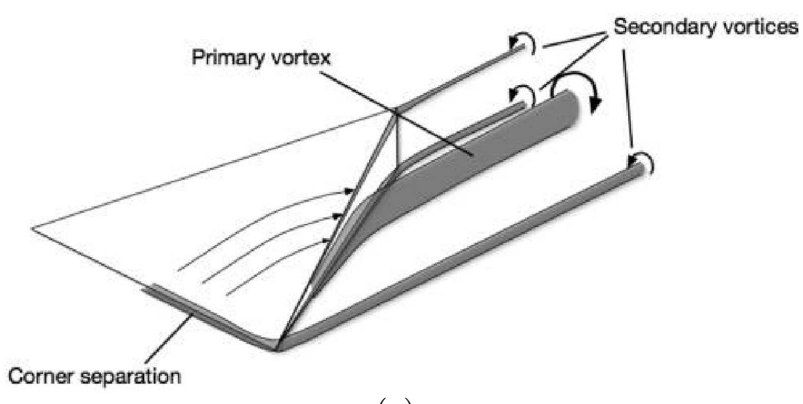

(a)

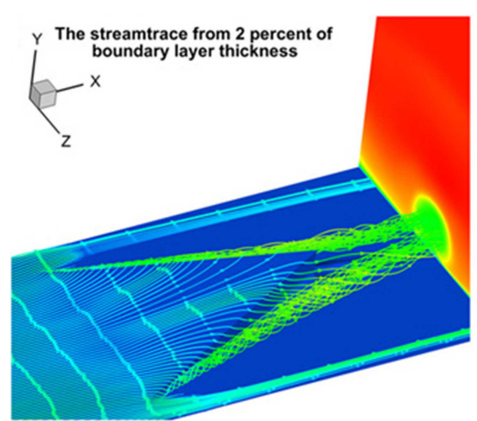

(b)

FIGURE 21. Micro-ramp flow features in a supersonic boundary layer.(a)sketch of vortices generated around micro-ramp (one side only for clarity) (Babinsky et al. 2009). (b)streamlines passing around the micro-ramp, originating from supersonic stream at wall parallel plane with 2 percent of boundary layer thickness (Wang et al. 2015a)

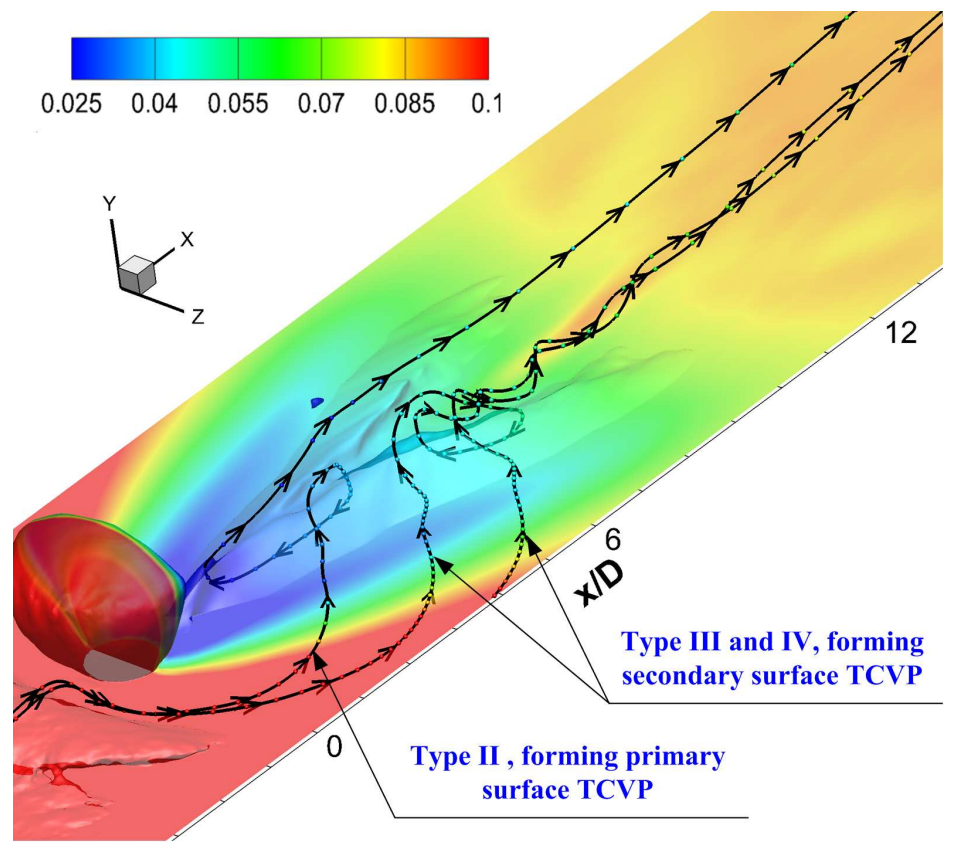

Figure 22. Flow topology (Type II, III and IV) demonstrated by streamlines in jet wake with transparent iso-surface of $u / U_{\infty}=0.0$ colored by local pressure of $J=5.5$ case

in the way as the micro-ramp described by Wang et al. (2015a). For figure 24(d), the streamlines cover a larger area in the separation wing than figure 24(b), which means that crossflow in the outer boundary layer enters into the bottom of the separation bubble in Type II pattern. In figure 24(d) it is found that some streamlines go over the separation bubble, run into the reattachment valley and move into farfield directly, which corresponds to the prediction in section 3.2. This also reflects that the flow running into the reattachment valley has different origins at different wall normal location.

To clearly demonstrate the surface TCVPs, iso-surface of streamwise vorticity is shown in figure $25(\mathrm{a})$ for $J=5.5$ case. Arrows with solid line points to the primary and secondary surface TCVPs respectively. It is identified that the surface TCVPs are located near the 


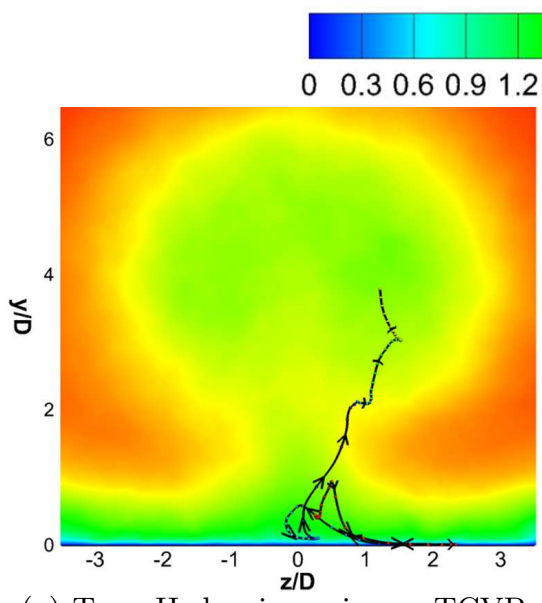

(a) Type II showing primary TCVP

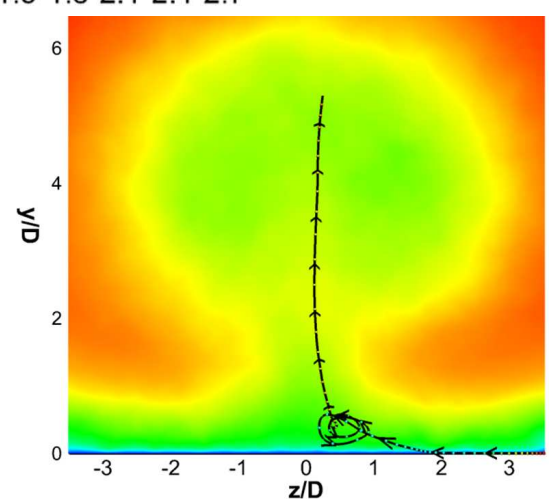

(b) Type III showing secondary TCVP

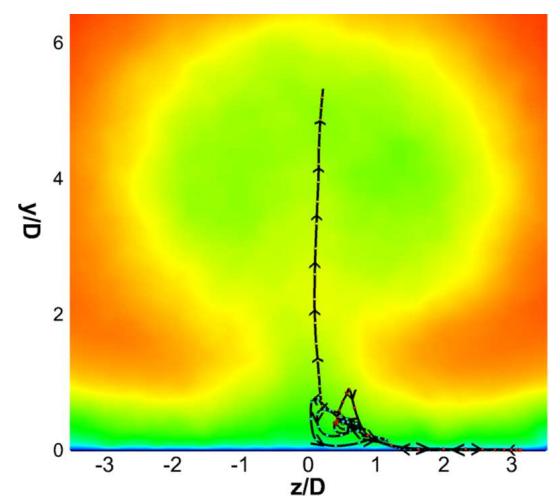

(c) Type IV showing a combined character of primary and secondary TCVP

FIgURE 23. Front view of typical streamlines of Type II, III and IV in jet wake with a Mach number slice at $x / D=40$ to demonstrate TCVPs of $J=5.5$

wall. It is seen that the surface TCVPs have an opposite rotating direction to the major CVP. The primary TCVP in the leeward separation zone has the same rotation direction with the secondary TCVP in reattachment zone. This is why usually it is not easy to distinguish them if $3 \mathrm{D}$ streamlines are not given. Separation line is imposed at $y / D=0.0$ slice to identify separation zone. It is shown that the secondary TCVP is separate from the primary TCVP in the jet lee, especially in the separation wing. It is therefore concluded that the primary TCVP is a single CVP which occurs in the herringbone recirculation and has no direct relation with the secondary TCVP.

Figure 25(b) shows a schematic of vortex tubes in the near-wall wake to demonstrate that primary TCVP and secondary TCVP. It is seen that primary TCVP is formed in the herringbone recirculation including jet leeward separation bubble and the oblique separation wing. Secondary TCVP is formed on the ramp leeward of the separation bubble adjacent to the reattachment valley. The separation bubble serves as a microramp vortex generator, which is well analyzed in a previous study Wang et al. (2015a). The inner boundary layer flow would roll and form the vortex tubes in the downstream field which corresponds to secondary TCVP. 

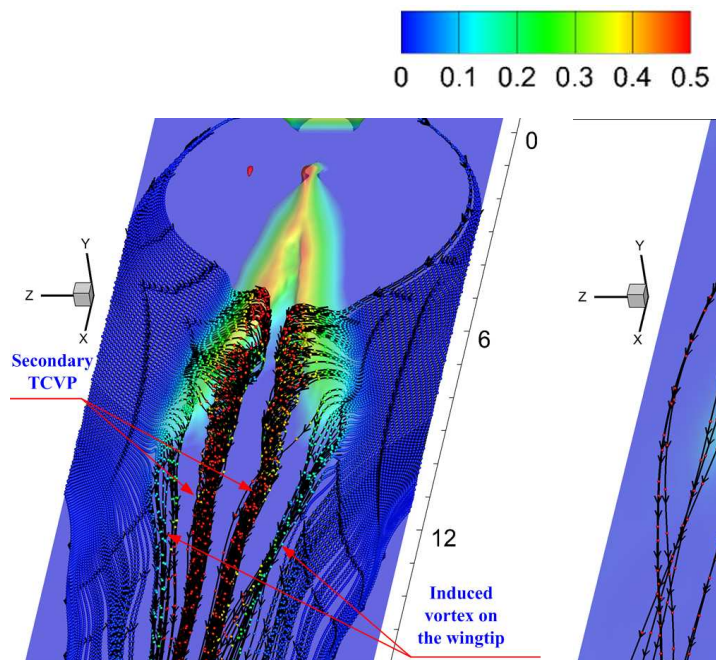

(a) iso-surface of $u / U_{\infty}=0.05$, Type III flow
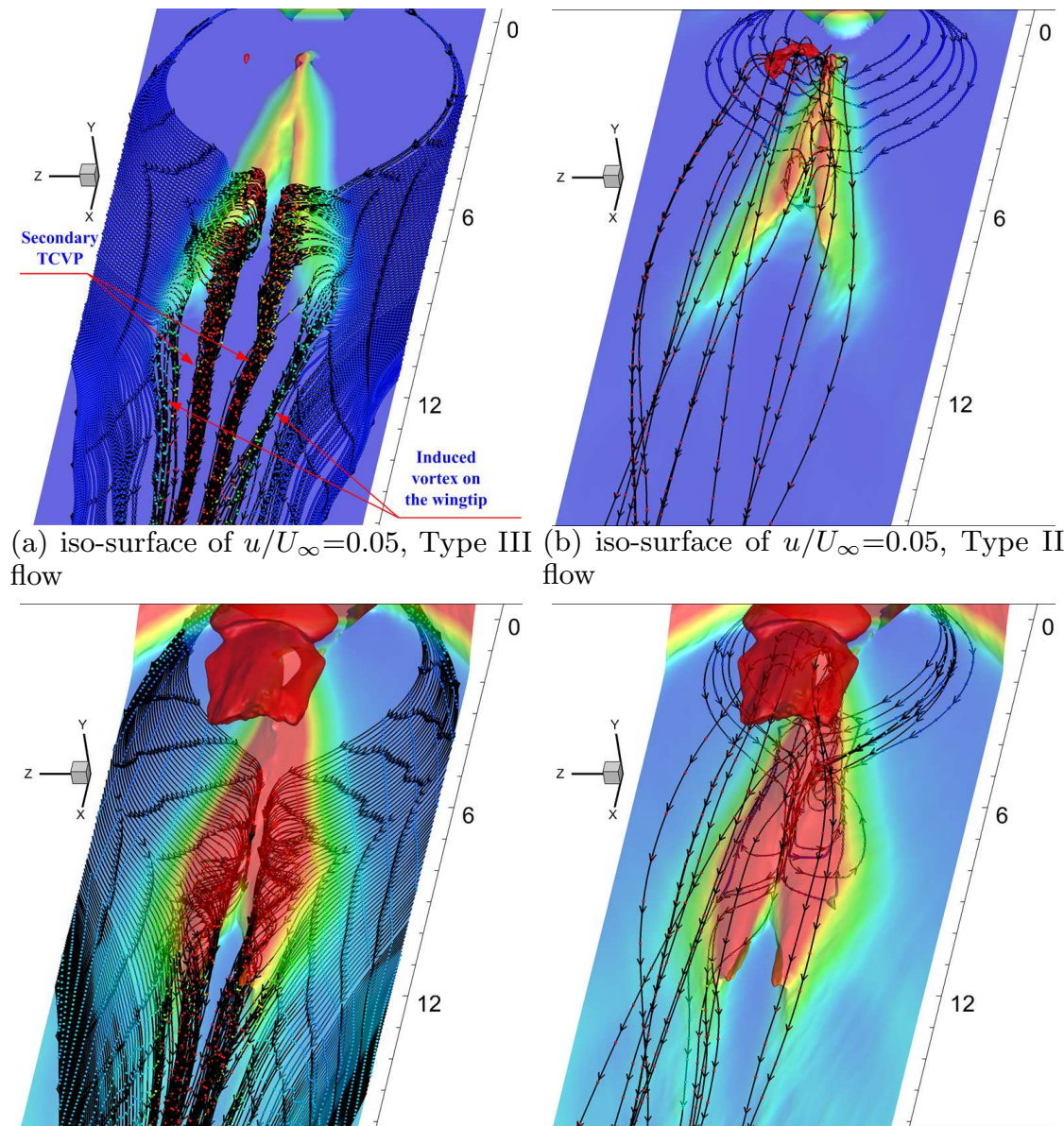
flow

(c) iso-surface of $u / U_{\infty}=0.5$, Type III

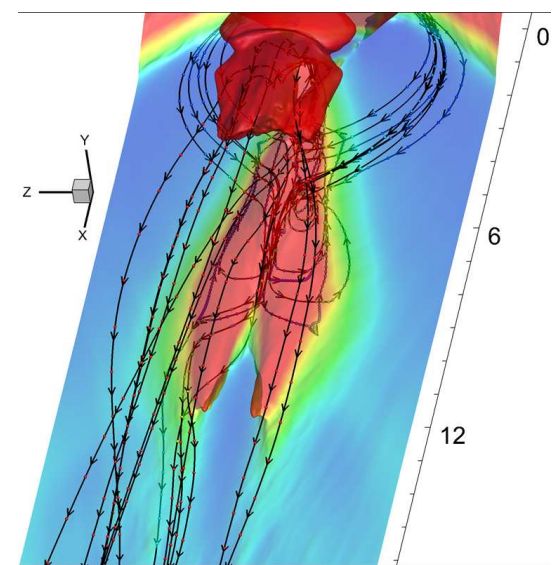
flow

flow

Figure 24. Streamlines passing the separation bubble (from an oblique back view), originating from lateral side on iso-surfaces of $u / U_{\infty}=0.05$ and $u / U_{\infty}=0.5$

Based on the numerical results and the analysis, it is concluded that the flow in the jet wake have very complex 3D structures including the collision shock, herringbone separation, reattachment valley, and two different TCVPs which are generated by different mechanisms in the near-wall jet wake. 


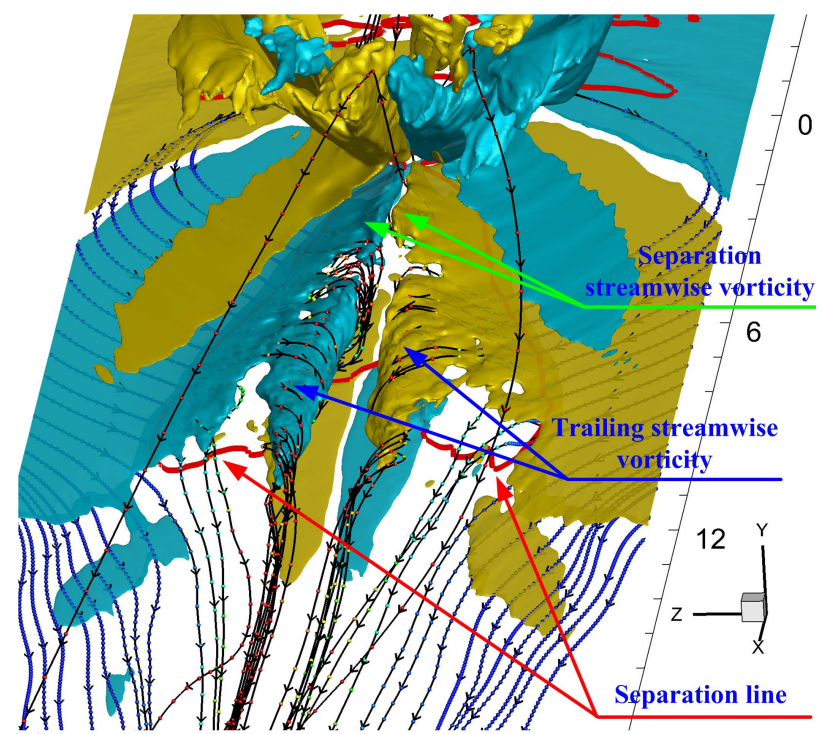

(a) iso-surface of streamwise vorticity $\Omega_{x}=-5$ and 5 , nondimensionalized by freestream velocity and jet orifice diameter

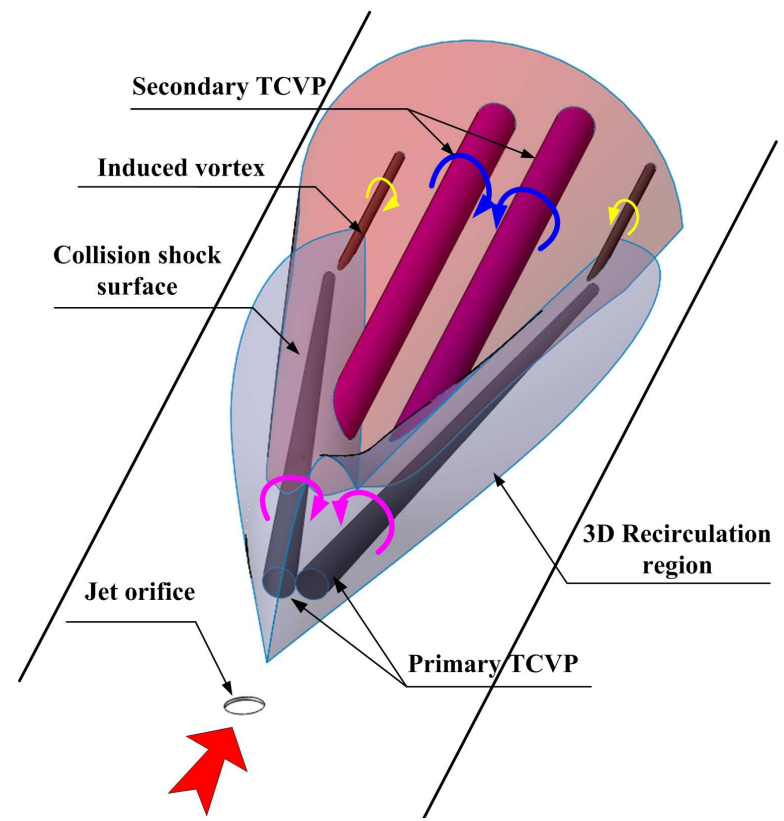

(b) schematic of vortex tubes (primary and secondary TCVPs) in jet wake

FIgURE 25. Iso-surface of streamwise vorticity and schematic of vortex tubes (primary and secondary surface TCVPs) in jet wakes.

\section{Conclusions}

In the present study, direct numerical simulations are conducted to uncover physical aspects of a transverse air jet injected into a supersonic air crossflow at a Mach number of 2.7. Simulations are carried out for two different jet-to-crossflow momentum flux ratios $(J)$ of 2.3 and 5.5. The simulations provide the instantaneous and time-averaged flow 
features around the transverse jet in the supersonic boundary layer. The results are as follows:

(1) The collision shock waves, the reattached shock waves and the major CVP and TCVP behind the jet are well captured. The collision shock waves induce a herringbone trailing separation region in the near-wall jet wakes. Analysis on velocity profiles along the streamwise direction and streamlines on wall parallel planes indicates existence of flow reattachment and multiple TCVPs in the near-wall jet wakes.

(2) Iso-surfaces of the streamwise velocity demonstrate that a reattachment valley exists in the herringbone recirculation zone near the symmetric plane. Flow in the separation region recirculates against the freestream direction, and forms the primary pair of counter-rotating vortices in the spanwise direction. The herringbone separation zone disappears in the farfield due to the interaction of the lateral supersonic flow around the jet and the reattached expansion flow in the reattachment valley.

(3) Streamlines running across the reattachment valley in the jet wake exhibit four types of flow patterns. For Type I, streamlines go around the jet barrel, converge in the jet lee and rise up following the suction by the major CVP. For Type II, streamlines go over the separation into the reattachment valley and concentrate in the vicinity upstream of the reattachment point. Furthermore, these streamlines recirculate and rotate following the oblique separation wing which acts as a tunnel which streamlines penetrate and swirl in. This recirculating flow corresponds to the primary surface TCVP. The recirculated streamlines concentrate in the jet lee and rise up due to the major CVP. For Type III, the wing of the herringbone recirculation acts as a micro-ramp vortex generator, and streamlines run across the separation wing and rotate to form a swirling vortex rope further downstream. This swirling flow corresponds to the secondary TCVP, which is different from the primary TCVP formed in Type II pattern. For Type IV, streamlines go cross the separation wing, are pushed by the reattachment compression and recirculate through the oblique separation wing. Furthermore, these streamlines run into the reattachment valley again and follow the secondary TCVP to move downstream. Streamlines in Type III and IV rotate and lose the swirling strength downstream of the reattachment valley due to the vanishing of the herringbone separation and the local dissipation, which corresponds to the termination of the secondary TCVP.

(4) Based on the numerical results, a 3D schematic of flow features in the jet wake is presented to explain surface TCVP formations. The primary TCVP is formed in the herringbone recirculation zone, while the secondary TCVP is formed in the reattachment valley and the separation bubble serves as a micro-ramp vortex generator.

\section{Acknowledgement}

This work is funded by the National Science Foundation of China (grants: 11472305 and 11522222) and outstanding youth fund of National University of Defense Technology. Sincere thanks to Professor N. D. Sandham of the University of Southampton for providing the SBLI(shock-/boundary-layer interaction) DNS(Direct Numerical Simulation) code and the helpful discussion on data analysis. Supercomputer time provided by the UK Engineering and Physical Sciences Research Council under the project Thermal and Reactive Flow Simulation on High-End Computers (Grant No. EP/J016381/1) is gratefully acknowledged. 
Babinsky, H., Li, Y. \& Ford, C. W. P. 2009 Microramp control of supersonic oblique shockwave/boundary-layer interactions. AIAA $J$ 47(3), 668-675.

Ben-Yakar, A., Mungal, G. M. \& Hanson, R. K. 2006 Time evolution and mixing characteristics of hydrogen and ethylene transverse jets in supersonic crossflows. Phys. Fluids 18, 26-101.

Chai, X., Iyer, P. S. \& Mahesh, K. 2015 Numerical study of high speed jets in crossflow. J. Fluid Mech. 785, 152-188.

Dickmann, D. A. \& Lu, F. K. 2009 Shock/boundary-layer interaction effects on transverse jets in crossflow over a flat plate. J. Spacecr. Rockets 46(6), 1132-1141.

Duan, L., Beekman, I. \& Martin, M. P. 2011 Direct numerical simulation of hypersonic turbulent boundary layers. part 3. effect of mach number. J. Fluid Mech. 672, 245-267.

Duan, L., Choudhar, M. M. \& Wu, M. 2014 Numerical study of acoustic radiation due to a supersonic turbulent boundary layer. J. Fluid Mech 746, 165-192.

Ducros, F., Ferrand, V., Nicoud, F., Weber, C., Darracq, D., Gacherieu, C. \& Poinsot, T. 1999 Large-eddy simulation of the shock/turbulence interaction. J. Comput. Phys. 152, 517-549.

Everett, D. E., Woodmansee, M. A., Dutton, J. C. \& Morris, M. J. 1998 Wall pressure measurements for a sonic jet injected transversely into a supersonic crossflow. J. Propul. Power 14(6), 861-886.

Gaitonde, D. V. 2015 Progress in shock wave/boundary layer interactions. Progress in Aerospace Sciences 72, 80-99.

Gamba, M. \& Mungal, M. G. 2015 Ignition, flame structure and near-wall burning in transverse hydrogen jets in supersonic crossflow. J. Fluid Mech. 780, 226-273.

Gruber, M. R., Nejad, A. S., Chen, T. H. \& Dutton, J. C. 1997 Large structure convection velocity measurements in compressible transverse injection flowfields. Exp. Fluids 22, 397407.

Gruber, M. R., Nejad, A. S., Chen, T. H. \& Dutton, J. C. 2000 Transverse injection from circular and elliptic nozzles into a supersonic crossflow. J. Propul. Power 16(3), 449-457.

Hu, X. Y., WANG, Q. \& AdAms, N. A. 2010 An adaptive central-upwind weighted essentially non-oscillatory scheme. J. Comput. Phys. 229, 8952-8965.

Jeong, J. \& Hussain, F. 1995 On the identication of a vortex. J. Fluid Mech 285, 69-94.

Karagozian, A. R. 2010 Transverse jets and their control. Prog. Energy Combust. Sci. 36(5), $531-553$.

KAWAI, S. \& LELE, S. K. 2010) Large-eddy simulation of jet mixing in supersonic crossflows. AIAA J. 48(9), 2063-2083.

Mahesh, K. 2013 The interaction of jets with crossflow. Annu. Rev. Fluid Mech. 45, 379-407.

Morkovin, M. V. 1962 Effects of compressibility on turbulent flows. In In Mecanique de la Turbulence (ed. A. Favre), pp. 367-380. CNRS.

Morkovin, M. V., Pierce, C. A. J. \& Craven, C. E. 1952 Interaction of a side jet with a supersonic main stream. Tech. Rep. 35. Eng. Res. Inst., Univ. Michigan.

Muppidi, S. \& MAHESh, K. 2005 Study of trajectories of jets in crossflow using direct numerical simulations. J. Fluid Mech 530, 81-100.

Portz, R. \& Segal, C. 2006 Penetration of gaseous jets in supersonic flows. AIAA J 44(10), $2426-2429$.

Rana, Z. A., Thornber, B. \& Drikakis, D. 2011 Transverse jet injection into a supersonic turbulent cross-flow. Phys. Fluids 23 (046103), 1-22.

Rothstein, A. D. \& Wantuck, P. J. 1992 A study of the normal injection of hydrogen into a heated supersonic flow using planar laser-induced fluorescence. In AIAA92-3423.

SAndhAm, N. D. 2016 Effects of compressibility and shock-wave interactions on turbulent shear flows. Flow Turbul. Combust. 97, 1-25.

Sandham, N. D., Johnstone, R. \& Jacobs, C. T. 2017 Surface-sampled simulations of turbulent flow at high reynolds number. Int $J$ Numer Meth $\mathrm{Fl} \mathbf{8 5}, 1-13$.

Sandham, N. D., Li, Q. \& Yee, H. C. 2002 Entropy splitting for high-order numercial simulation of compressible turbulence. J. Comput. Phys. 178, 307-322.

Sandham, N. D., Schlein, E., Wagner, A., Willems, S. \& Steelant, J. 2014 Transitional shock-wave/boundary-layer interactions in hypersonic flow. J. Fluid Mech. 752, 349-382. 
Sandhu, H. S. \& Sandham, N. D. 1996 Simulations of leading-edge receptivity to free-stream disturbances. Tech. Rep.. Faculty of Engineering, Queen Mary Westfield College.

Santiago, J. G. \& Dutton, J. C. 1997 Velocity measurements of a jet injected into a supersonic crossflow. J. Propul. Power 13(2), 264-273.

Schetz, J. \& Billig, F. S. 1966 Penetration of gaseous jets injected into a supersonic stream. J.Spacecr.Rockets 3, 1658-1665.

Schlatter, P. \& Orlu, R. 2010 Assessment of direct numerical simulation data of turbulent boundary layers. J. Fluid. Mech. 659, 116-126.

Sun, M. B. \& Hu, Z. W. 2018 Generation of upper trailing counter-rotating vortices of a sonic jet in a supersonic crossflow. AIAA $J \mathbf{5 6}, 1047-1059$.

Sun, M. B., Zhang, S. P., Zhao, Y. H., ZhaO, Y. X. \& Liang, J. H. 2013 Experimental investigation on transverse jet penetration into a supersonic turbulent crossflow. Sci. China Tech. Sci. 56(8), 1989-1998.

Thompson, K. W. 1987 Time dependent boundary conditions for hyperbolic systems. J. Comput. Phys. 68, 1-24.

Touber, E. 2010 Unsteadiness in shock wave boundary layer interactions. PhD thesis, University of Southampton.

Touber, E. \& Sandham, N. D. 2009 Large-eddy simulation of low-frequency unsteadiness in a turbulent shock-induced separation bubble. Theor. Comput. Fluid Dyn. 23, 79-107.

Viti, V., Neel, R. \& Schetz, J. A. 2009 Detailed flow physics of the supersonic jet interaction flow field. Phys. Fluids 21 (4), 046101.

WANG, B., Liu, W. D., Sun, M. B. \& ZhaO, Y. X. 2015a Fluid redistribution in the turbulent boundary layer under the microramp control. AIAA J. 53 (12), 3775-3787.

Wang, B., Sandham, N. D., Hu, Z. \& W, Liu. 2015b Numerical study of oblique shockwave/boundary-layer interaction considering sidewall effect. J. Fluid Mech. 767, 526-561.

WAng, H. B., WANG, Z. G., Sun, M. B. \& QIN, N. 2013 Hybrid reynolds-averaged navierstokes/large-eddy simulation of jet mixing in a supersonic crossflow. Sci. China Tech. Sci. 56(6), 1435-1448.

Won, S.-H., Jeung, I.-S., PArent, B. \& Choi, J.-Y. 2010 Numerical investigation of transverse hydrogen jet into supersonic crossflow using detached-eddy simulation. AIAA J. 48(6), 1047-1058.

XIE, Z. T. \& CASTro, I. P. 2008 Efficient generation of inflow conditions for large-eddy simulation of street-scale flows. Flow Turbul. Combust. 81, 449-470.

Yee, H. C., Sandham, N. D. \& Djomehri, M. J. 1999 Low-dissipative high-order shockcapturing methods using characteristic-based filters. J. Comput. Phys. 150, 199-238.

Zhang, Y. J., Liu, W. D. \& Sun, M. B. 2016 Effect of microramp on transverse jet in supersonic crossflow. AIAA J. 54(12), 4041-4044. 